\title{
STS Protects Diabetic Glomerular Vascular Endothelial Barrier by Ameliorating EPC Dysfunction: Targeting RAGE-TXNIP-NLRP3 Inflammasome Pathway
}

\author{
Yan-Yan Heng \\ Changzhi Medical College Affiliated Heping Hospital \\ Xiao-Yan Zhang \\ Changzhi Medical College Affiliated Heping Hospital \\ Fei-Fei Wang \\ Changzhi Medical College Affiliated Heping Hospital \\ Peng-Fei Zhang \\ Changzhi Medical College Affiliated Heping Hospital \\ wei wei ( $\boldsymbol{\nabla}$ jaywei@czmc.edu.cn ) \\ changzhi medical college
}

\section{Research}

Keywords: Diabetic Kidney Disease, Endothelial Progenitor Cells, Sodium Tanshinone Sulfonate $₫ A$, NLRP3 inflammasome, Caspase-1

Posted Date: October 29th, 2021

DOI: https://doi.org/10.21203/rs.3.rs-988095/v1

License: (c) (i) This work is licensed under a Creative Commons Attribution 4.0 International License.

Read Full License 


\section{Abstract}

Background: Glomerular endothelial cell (GEC) injury is one of the crucial causes of diabetic kidney disease (DKD). Endothelial progenitor cell (EPC) is the essential mechanism of vascular endothelial repair, which damages by diabetic pathology. Sodium Tanshinone Sulfonate $\mathbb{\text { A }}$ (STS) is known to protect endothelium, but the mechanism and the role in DKD need to be studied.

Methods: EPC was treated with high glucose $(\mathrm{HG})$, and thioredoxin interacting protein (TXNIP), NLR family pyrin domain containing 3 (NLRP3) inflammasome, DNA damage, proliferation, differentiation and senescence were detected; STS and EPC were intravenous injected into diabetic nude mice, the urine protein quantitation and urine protein/creatinine were detected; the Dil-labeled EPC was traced and the expression of TXNIP, caspase-1 (p20), p21, Ki67, CD31 were detected by fluorescence co-location in glomerulus.

Results: We found that STS inhibited HG-induced TXNIP expression and NLRP3 inflammasome activation, catalase (CAT) inactivation, DNA damage, senescence区 STS restored EPC proliferation and differentiation functions; advanced glycation end products (AGEs) produced in HG treated EPC supernatant, the receptor of AGE (RAGE) blocking inhibited TXNIP expression and NLRP3 inflammasome activation, which mimicked by STS. STS protected EPC functions in diabetic glomerular and enhanced EPC renal function amelioration.

Conclusions: We concluded that STS watched CAT activity to prevent HG-induced EPC DNA damage, proliferation, differentiation dysfunction, accelerated senescence by inhibiting the RAGE-TXNIP-NLRP3 inflammasome-caspase-1 pathway.

\section{Background}

Diabetic kidney disease (DKD) is one of the major microvascular complications of diabetes and the leading cause of the end-stage renal disease (ESRD)[1]. The main symptom of early DKD is persistent microalbuminuria[2]. Proteinuria may be associated with glomerular filtration barrier (GFB) injury. GFB simply divides into three layers: glomerular endothelial cell layer (GEC), glomerular basement membrane, podocyte layer[3]. As the first GFB barrier directly contacts with blood, it has been proved that the glomerular endothelium (GE) cells secreted vasoactive substances and played an essential role in maintaining the glomerular homeostasis and the dysfunction of GE was a marker of the early progression of DKD, leading to the damage of GFB and the formation of proteinuria[4]. GEC fenestrations are trans-cytoplasmic holes, which accounts for $20 \%-50 \%$ of the surface area of the endothelial cell layer, and the diameter of the endothelial pores is about $60-100 \mathrm{~nm}$, which is 15 times more than the diameter of the albumin molecule (3.6nm)[5]. The negatively charged proteins synthesized and secreted by GEC combine with glycosaminoglycan in covalent bonds to form a networked layer of the glomerular endothelial surface layer (ESL) to fill the GEC hole to prevent leakage of albumin[6]. GEC loss may cause 
GFB dysfunction and DKD progress[7, 8]. Therefore, maintaining the health of GEC or GE renewing after injury may be the feasible therapy of DKD.

Endothelial progenitor cell (EPC) is one of the bone marrow (BM) source stem cells, early-EPC is mainly characterized by positive surface antigen markers such as CD34, CD133 and VEGFR2, CD133 was decreased in the late-EPC, and the proliferation ability is increased, at the same time late-EPC presented the physiological functions of repairing vascular endothelial injury and angiogenesis[9-11]. It is currently believed that following the injure, EPC is mobilized from BM, recruited to the injured site, differentiated into the mature endothelial cell (EC) or vascular smooth muscle cell (VSMC), simultaneously, EPC secreted a variety of cytokines to promote the remodeling of existing vessels or participate in the assembly of new vessels[12-14]. More, endothelium repair and angiogenesis progress can be enhanced by EPC paracrine[15, 16]. However, it was reported that diabetic status impaired endothelial repair function, EPC mobilization was weakened, apoptosis and oxidative stress were increased, and the senescence was accelerated[17, 18]. These conclusions indicated that the repair function after the vascular endothelial injury was weakened in the pathological environment of diabetes, leading to the EC loss could not be repaired in time. But the mechanism of EPC repair endothelium dysfunction in diabetic pathology and its role in the progression of DKD remain unclear.

Tanshinone IIA is one of the most lipophilic pharmacologically active ingredients extracted from the root of Traditional Chinese Medicine Salvia miltiorrhiza[19]. Its sodium sulfonate products Sodium Tanshinone Sulfonate $\mathbb{\triangle A}$ (STS) is the water-soluble modification. Because of its anti-inflammatory, antioxidative, and anti-apoptotic pharmacological effects, STS is a widely recognized clinical drug for treating cardiovascular diseases[20-22]. Recent researches have shown that STS inhibited collagen deposition in the glomerular basement membrane of diabetic animals by regulating the PERK pathway to alleviate endoplasmic reticulum stress[23], and transcriptome analysis of mesangial cells in animals with diabetic nephropathy showed that STS had a significant protective effect on DKD[24]. It can be seen that TS may have a protective effect on the progression of DKD, but its protective effect on diabetic glomerulopathy remains to be studied.

Moreover, the endothelial-protective effects of STS have been reported widely. STS protected the endothelial barrier function of the cardiovascular by preventing apoptosis of endothelial cells, oxidative and ischemia-reperfusion injury of endothelial cells[25,26]. However, the protective effect of TS on glomerular endothelial cells has not been reported. Some studies have revealed that by inhibiting tumor necrosis factor-a (TNF-a), TS can down-regulate the expression of VCAM- 1 and ICAM- 1 in EPC, and improve the damaged cell functions of EPC such as proliferation, migration, adhesion and tubular formation, which has a potential pharmacological effect on preventing atherosclerosis[27, 28]. Therefore, STS may protect EPC function, but whether it can restore the function of diabetic EPC in the glomerular region to repair the glomerular microvascular endothelial barrier remains need to be further studied.

This study aims to observe the repair of glomerular endothelial barrier damage in diabetes mellitus by EPC, slow down the progression of DKD, and partially reveal the mechanism of functional impairment of 
EPC induced by the high glucose environment. The present study demonstrated that excess glucose might form advanced glycation end products (AGEs). It activated the receptor of the advanced glycation end product (RAGE)-thioredoxin-interacting protein (TXNIP)-NLR family pyrin domain containing 3 (NLRP3) inflammasome pathway to eliminate catalase (CAT) activity and cause EPC dysfunction. STS restored the proliferation and differentiation function of EPC and slowed down its aging by reducing TXNIP expression to inhibit the NLRP3 inflammasome-caspase-1 pathway-dependent CAT inactivation in diabetes.

\section{Methods}

\subsection{Isolation, culture, and characterization of mouse bone marrow-derived EPC}

EPCs were isolated from mouse bone marrow according to the previous study[29]. Bone marrow medulla was centrifuged by Density Gradient with a Human peripheral blood lymphocyte isolation fluid (Ficoll Plus 1.077, Solarbio, P4350, Beijing China) to obtain the bone marrow Mononuclear Cells (MNCs). MNCs were plated in fibronectin (Solarbio, F8180, Beijing, China) coated cell culture bottle with a ventilation filter and maintained in Endothelial Growth Medium-2 (EGM-2, Lonza, CC-4176, USA) with 10\% fetal bovine serum (FBS, EPHRAIM, 26-500-FBS China) in a cell incubator with $37^{\circ} \mathrm{C}$ and $5 \% \mathrm{CO}_{2}$. The medium was replaced every $48 \mathrm{~h}$. Cell colonies those appeared about the first 7 days later, these cells had a fusiform morphology and were defined as the early EPC[30]. After 28 days of culture, cells emerged abilities of proliferation and tube formation, had a pebble-like compact arrangement as endothelial cells (EC), and were called endothelial colony-forming cell (ECFC), outgrowth endothelial cells (OECs), or late-EPC[31].

There is no consensus about EPC cell typing now, but the most recognized characterizations of EPC are CD34, CD133, and VEGFR2 positive and Human Dil-Acetylated Low-Density Lipoprotein (Dil-Ac-LDL) and FITC-UEA-I double-positive[32]. The cells cultured about 28 days were plated on sterilized coverslips (Beyotime FCGF18, shanghai, China) and cultured in the medium with $5 \mu \mathrm{g} / \mathrm{ml}$ Human Dil-Ac-LDL (Maokangbio, MP6013, Shanghai, China) at $37^{\circ} \mathrm{C}$ for $4 \mathrm{~h}, 4 \%$ paraformaldehyde (PFA) fixed $30 \mathrm{~min}$, $10 \mu \mathrm{g} / \mathrm{ml}$ FITC-UEA-I (Maokangbio, MP6038, Shanghai, China) incubated for $1 \mathrm{~h}$ at room temperature, washed by PBS for three times and then the fluorescence images were captured by a fluorescence microscope (Carl Zeiss,Axio Scope A1, Germany). The fixed cells on coverslips were blocked with $5 \%$ bovine serum albumin (BSA Solarbio, A8010, Beijing, China) for 1.5h The primary antibodies, rabbit antiCD34 (1:100, Wanleibio, WL02529, Shenyang, China), rabbit anti-CD133 (1:100, Proteintech, 66666-1-lg, Wuhan China) and rabbit anti-VEGFR2 (1:100, Wanleibio, WL02294, Shenyang, China) incubated overnight at $4^{\circ} \mathrm{C} \square$ the second antibody (Cy3-conjugated Goat Anti-rabbit IgG H+Lه1:100هProteintech, Wuhan China SA00009-2) incubated for $2 \mathrm{~h}$ at room temperature in the dark. After being washed by PBS, the images were captured by a fluorescence microscope (Carl Zeiss, Axio Scope A1, Germany). The mouse aorta vascular endothelial cells (MAVECs) as a negative control.

\subsection{Animals}


Adult male BALB/c nude mice, weighing 20-25g, were obtained from Gempharmatech, Nanjing, China.

High-fat diet (HFD, $10 \%$ saccharose, $10 \%$ lard, $10 \%$ sugar, $5 \%$ egg yolk powder, $0.5 \%$ cholesterol, $64.5 \%$ basal chow) and a low dose of streptozotocin (STZ) to develop diabetes according to the method described previously[33]. Briefly, after 4 weeks of feeding with HFD, mice were administered STZ ( $65 \mathrm{mg} / \mathrm{kg}$ body weight, $\mathrm{pH} 4.5$ ) or citrate buffer (vehicle) by intraperitoneal (i.p.) injections once a day for 5 days. After one week, the mice fasted for $12 \mathrm{~h}$ for fasting blood glucose (FBG) testing. Plasma glucose concentration was detected using a commercial glucometer (GA-3, Sinocare, Changsha, China). Fasted mice with blood glucose levels higher than $11.1 \mathrm{mmol} / \mathrm{L}$ were considered as diabetic mice. 24h urinary albumin quantitation and plasma and urinary creatinine quantification and urinary albumin-to-creatinine ratio were used to evaluate renal function in diabetic mice. Urinary albumin and creatinine were detected by a commercial kit purchased from Jiancheng bioengineering institute (C035-2-1 and C011-2-1 Nanjing, China)

\subsection{HE and PAS staining of the glomerulus}

Mouse kidney was perfused by PBS and fixed in 4\% PFA, dehydrated and embedded in paraffin. Paraffin sections were cut at $5 \mu \mathrm{m}$. After deparaffinization and rehydration, glomerulus sections were dyed by hematoxylin solution (hematoxylin $1 \mathrm{~g}$, sodium iodate $0.2 \mathrm{~g}$, aluminum potassium sulfate, $50 \mathrm{~g}$, citric acid $1 \mathrm{~g}$, chloral hydrate $50 \mathrm{~g}$, distilled water added to 1 liter) for 10 minutes, alcoholic eosin $(2.5 \mathrm{~g}$ eosin, $500 \mathrm{ml}$ distilled water, hydrochloric acid $10 \mathrm{ml}$, filter product dissolved in $1000 \mathrm{ml} 95 \%$ alcohol, double diluted before use) for 30 seconds respectively.

Periodic Acid-Schiff stain (PAS) of glomeruli was performed using the PAS stain kit (Shanghai Yuanye Bio-Technology Co., Ltd, Shanghai, China). After deparaffinization and rehydration, glomerulus sections were immersed in periodic acid for $5 \mathrm{~min}$, washed by running water and distilled water respectively, then in Schiff reagent for $10 \mathrm{~min}$ in the dark. After being washed with running water for $10 \mathrm{~min}$, the nuclear was stained by the hematoxylin solution. The sections were washed with running water until the nuclear turning blue.

The sections were dehydrated by the gradient of ethanol, and graphs were captured by microscope (Carl Zeiss, Axio Scope A1, Germany) in the light field mode.

\subsection{Western Blot Analysis and co-immunoprecipitation}

Western blot analysis was performed as described previously[34]. Briefly, cell total protein and membrane protein samples were extracted using Membrane and Cytosol Protein Extraction Kit (Beyotime, P0033, Shanghai, China), nuclear protein samples were extracted using Nuclear and Cytoplasmic Protein Extraction Kit (Beyotime, P0027, Shanghai, China). Protein concentration was quantified using a BCA Protein Assay Kit (Keygentec, KGP903, Nanjing, China). According to the quantification, protein samples were adjusted to equal with $5 \times$ loading buffer (Beyotime, P0015, Shanghai, China), and samples were boiled for 5 minutes at $95^{\circ} \mathrm{C}$. Equal amounts of cell lysate protein were resolved by SDS-PAGE using $5 \%$ $(\mathrm{w} / \mathrm{v})$ stacking and $8 \%-15 \%(\mathrm{w} / \mathrm{v})$ separating polyacrylamide gels, and then transferred to $0.45 \mu \mathrm{m}$ 
polyvinylidene difluoride membrane (PVDF, Millipore, USA), which were then blocked for 1.5 hours in $5 \%$ $(\mathrm{w} / \mathrm{v})$ non-fat milk diluted in Tris-buffered saline (TBST, $100 \mathrm{mM}$ Tris-HCL, $\mathrm{pH}$ 7.4) with $0.01 \%(\mathrm{v} / \mathrm{v})$ Tween-20, and incubated with the primary antibodies overnight at $4^{\circ} \mathrm{C}$. The primary antibodies used were rabbit anti-TXNIP (1:500, Beyotime AF8277, Shanghai, China), rabbit anti-NLRP3 (1:1000, Abways Technology, Inc., CY5651, Shanghai, China), rabbit anti-p20 (1:500, Abways Technology, Inc., AY0406 Shanghai, China), rabbit anti-Ki67 (1:500, Wanleibio, WL01384a, Shenyang, China), rabbit anti-IL-1 $\beta$ (1:1000,Wanleibio, WL00891, Shenyang, China), rabbit anti- Histone H2A.X (1:500, Wanleibio, WL00616a, Shenyang, China), rabbit anti-CD31 (1:500, affinity, P16284, Changzhou, China), rabbit anti-VWF (1:1000, affinity, AF3000, Changzhou, China), rabbit anti-pan-cadherin (1:1000, Wanleibio, WL03295, Shenyang, China), rabbit anti-a-tubulin (1:4000, Abways, AB0048, Shanghai, China) $\square$ rabbit anti-Histone H3 (1:1000, Wanleibio, WL0984a, Shenyang, China). After being washed for 3 times, the membranes were incubated with goat anti-rabbit IgG (1:5000, Abways Technology, Inc., Shanghai, China) for $1.5 \mathrm{~h}$. The blot was detected by an automatic chemiluminescence/fluorescence image analysis system ( 5200 Multi, Tanon, Shanghai, China) with electrochemiluminescence (ECL, Tanon, Shanghai, China). Densitometric analysis of the images was performed with Image $\mathrm{J}$ software ( $\mathrm{NIH}$, Littleton, $\mathrm{CO}, \mathrm{USA})$.

\section{Co-immunoprecipitation (co-IP)}

$2 \times 10^{7}$ treated cells were lysed and extracted total protein using RIPA Lysis Buffer (Beyotime, P0013B, Shanghai, China). $1 \mu$ g Rabbit IgG (Bioworld technology, co, Ltd, Nanjing, China) and 20 $\mu$ Protein A+G Agarose (Beyotime, P2055, Shanghai, China) were added to each sample to remove the nonspecific binding. The primary rabbit anti-catalase antibody (Abways, CY6783, Shanghai, China) was added and combined with catalase, and samples were shocked slowly overnight at $4^{\circ} \mathrm{C}$. The immunoprecipitation was performed using $30 \mu \mathrm{l}$ Protein $A+G$ Agarose each sample for $2 \mathrm{~h}, 4^{\circ} \mathrm{C}$. After $1000 \mathrm{~g}$ centrifugation, the supernatant was discarded, the precipitate was suspended with 1×SDS loading buffer (Beyotime, P0015A, Shanghai, China), and boiled at $100^{\circ} \mathrm{C}$ for $3 \mathrm{~min}$. Samples were separated by SDS-PAGE gel, and CAT-p20 conjunction was detected by rabbit anti-p20 antibody (Wanleibio, WL02996a, Shenyang, China). As same as these steps, the p20-CAT conjunction was detected by rabbit anti-catalase antibody (Abways, CY6783, Shanghai, China).

\subsection{Catalase activity detection}

The activity of catalase was detected by in-gel catalase stain according to the previous study[35]. Cells were lysed by RIPA lysis buffer (Beyotime, P0013B, Shanghai, China) with PMSF (Beyotime, ST506, Shanghai, China) on ice, and the extract was centrifuged for $10 \mathrm{~min}$ at $4{ }^{\circ} \mathrm{C}$ and $10000 \mathrm{~g}$. The supernatant was mixed with non-reducing $4 \times$ SDS loading buffer $(50 \mathrm{mM}$ Tris- $\mathrm{HCl}, \mathrm{pH} 6.8,2 \%$ SDS, 10\% glycerol, 12.5 mM EDTA, $0.02 \%$ bromophenol blue) and electrophoresed on a 10\% PAGE gel in electrophoresis buffer (3.025g tris, $14.4 \mathrm{glycine}$ dissolve in $1 \mathrm{~L}$ deionized water). The gel was washed with deionized water three times and then immersed in $0.003 \% \mathrm{H}_{2} \mathrm{O}_{2}\left(2 \mu \mathrm{l} 30 \% \mathrm{H}_{2} \mathrm{O}_{2}\right.$ added to $20 \mathrm{ml}$ deionized water) for $10 \mathrm{~min}$ and stained with a freshly staining solution ( $2 \%$ potassium ferricyanide and $2 \%$ ferric chloride) until the bright 
band appeared. After three times washed by running water, the gels were photographed by an automatic chemiluminescence/fluorescence image analysis system (5200 Multi, Tanon, Shanghai, China). The atubulin detected by western blot was used to normalize the total protein.

\subsection{Immunofluorescence Analysis}

\section{Cell experiments}

EPCs ( $5 \times 10^{4}$ cells) were seeded on sterilized coverslips. After treatment, cells were washed three times by PBS $\left(137 \mathrm{mM} \mathrm{NaCl}, 2.7 \mathrm{mM} \mathrm{KCl}, 10 \mathrm{mM} \mathrm{Na}_{2} \mathrm{HPO}_{4}, 1.8 \mathrm{mM} \mathrm{KH}_{2} \mathrm{PO}_{4}\right)$ and fixed by $4 \%$ PFA for $30 \mathrm{~min}$. The cell membrane was perforated by $0.3 \%$ Triton X-100 (Biosharp, BS084, Hefei, China) for $15 \mathrm{~min}$ and the cell was blocked with $5 \%$ bovine serum albumin (BSA, Solarbio, A8010, Beijing, China) for 1.5 hours. The primary antibodies, rabbit anti-NLRP3 (1:200, Abways Technology, Inc., CY5651, Shanghai, China) and rabbit anti-PYCARD (ASC, 1:100, Abways Technology, Inc., AY0406, Shanghai, China), were incubated for 5 hours, respectively. After triple washed by PBS, NLRP3 and ASC were labeled by the second fluorescent antibodies Cy3-conjugated Affinipure Goat Anti-Rabbit lgG(H+L) (1:100, Proteintech, SA00009-2, Wuhan, China) and Goat Anti-Rabbit lgG (H+L) Alexa Fluor 488 (1:100, Abways Technology, Inc., AB0141, Shanghai, China) for 2 hours, respectively.

\section{Animal experiments}

Diabetic nude mice received Sodium tanshinone IIA sulfonate (STS, $15 \mathrm{mg} / \mathrm{kg} / \mathrm{d}$ Solarbio, ST8030, Beijing, China), a water-soluble substance obtained by sulfonation of tanshinone IIA injection for 7days and once Dil (CM-Dil, 0.5 $\mu \mathrm{g} / \mu \mathrm{l}, \mathrm{HB} 171031$, Yeasen, Shanghai, China) labeled EPCs $\left(1 \times 10^{6}\right)$ transplantation via tail vein. After PBS and 4\% PFA perfusion, the diabetic kidneys were cut into tissue blocks. Optimal cutting temperature compound (OCT, CelIPath, SA00009-2, U.K.) embedded mouse kidney sections were cut at $6 \mu \mathrm{m}$ and permeabilized with $0.1 \%$ Triton X-100-PBS after washing the OCT. The sections were fixed and blocked as same as the EPCs procedure. The sections were incubated with the primary antibodies, rabbit anti-CD31 (1:100, affinity, P16284, Changzhou, China) and rabbit anti-catalase (1:100, Abways Technology, Inc., CY6783, Shanghai, China), anti-p21 (1:100, Wanleibio, WL0362 Shenyang, China) and rabbit anti-p20 (ASC, 1:100, Wanleibio, WL02996a, Shenyang, China), rabbit antiVDUP1 (TXNIP, 1:100, sc-271237, Santa Cruz, USA), followed by Alexa Fluor 488. images were photographed by a fluorescent microscope (Carl Zeiss, Axio Scope A1, Germany), and the images of glomerulus were processed by ZEN blue 2.3 software (Carl Zeiss, Germany). The Pearson's coefficient which represents col-localization was calculated by Image $\mathrm{J}$ software $(\mathrm{NIH}$, Littleton, $\mathrm{CO}, \mathrm{USA})$.

\subsection{Catalase mutant and HEK293T cell transfection}

Catalase (CAT) point mutants were performed by transfecting pcDNA3.1 plasmid which was purchased from GENEWIZ (Suzhou, China). By calculating the molecular weight of the CAT fragment, aspartic acid sites in D298, D307, and D226 were replaced with alanine, generated D298A, D307A, and D226A CAT 
plasmids, and the wild type CAT was also constructed into pcDNA3.1 plasmid. These mutant plasmids were delivered into EPCs by Lipofectamine ${ }^{\mathrm{TM}} 2000$ Transfection Reagent (Invitrogen, 11668019, USA) and HEK293T cells (Fenghbio, Changsha, China) by Calcium Phosphate Cell Transfection Kit (Beyotime, C0508, Shanghai, China). EPCs were treated by LG, HG, HG with LPS+ATP and WEHD, HEK293T cells were transfected by NLRP3, ASC, and caspase-1 plasmids those purchased from Fenghbio (Changsha, China), and the NLRP3 inflammasome was activated by LPS+ATP treatment. The protein samples extracted from EPCs and HEK293T cells were detected by western blot and co-IP.

\subsection{BrdU incorporation assay}

BrdU incorporation assay was performed as described previously[37]. EPCs were seeded on coverslips in six-well plates with $L G$ and $H G$ medium to $60-70 \%$ confluences and then incubated with serum-deprived LG and HG medium for $12 \mathrm{~h}$. EPCs were labeled with BrdU (10 $\mu \mathrm{M}$, Solarbio, Beijing, China) for $2 \mathrm{~h}$. After fixed by $4 \%$ PFA, perforated by $0.3 \%$ Triton $\mathrm{X}-100$ and denatured by $2 \mathrm{M} \mathrm{HCl}$, EPCs were incubated with mouse anti-BrdU primary antibody (1:100, Proteintech ${ }^{T M}$, Wuhan, China) overnight, followed by Cy3conjugated Goat Anti-mouse IgG $(H+L)\left(1: 100\right.$, Proteintech ${ }^{T M}$, Wuhan, China), nuclei were labeled by DAPI. The immuno-fluorescence images were taken by a Fluorescence microscope (Carl Zeiss, scope A1, Germany). The percentage of BrdU positive cells to the total amount of cells was calculated by Image $J$ software (NIH, Littleton, CO, USA).

\subsection{AGEs detection}

The abundance of AGEs in EPC supernatant and mice serum was detected using a commercial kit (ab273298, Abcam, USA). EPCs $\left(3 \times 10^{3} / \mathrm{ml}\right)$ were seeded in 96 well plate, cultured by LG (Ctrl), HG (10, 20, $35 \mathrm{mM})$ for $48 \mathrm{~h}$, and $\mathrm{HG}(35 \mathrm{mM})$ for $24,48,72 \mathrm{~h}$ respectively. Followed the procedure described in the specification, BSA as the negative control, BSA-AGE as the positive control, supernatant and serum samples were added into white flat-bottom 96-well plate (FCP968, Beyotime, Shanghai, China). Relative Fluorescence Units (RFU) were detected by a fluorescent microplate reader (Molecular Devices SpectraMax M4, USA) at Ex360nm/Em460nm. A standard curve was made using the BSA-AGE standard reagent $(10 \mathrm{mg} / \mathrm{ml})$. The relative abundance of AGEs in samples was calculated according to the standard curve.

\subsection{Hydrogen Peroxide detection}

Hydrogen Peroxide content in EPC was detected using a commercial kit (Solarbio, BC3595, Beijing,

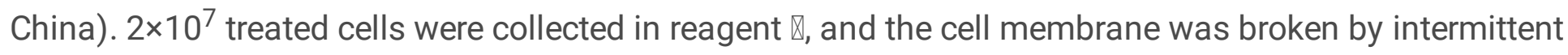
ultrasonic, cell debris was centrifuged at $8000 \mathrm{~g}$ for $10 \mathrm{~min}$, the supernatant was collected. According to specification, the absorbance at $415 \mathrm{~nm}$ was detected by a microplate reader (Molecular Devices SpectraMax M4, USA). The sample hydrogen peroxide content was calculated according to the absorbance of the standard substance with known concentration.

\subsection{EPC SA- $\beta$-gal staining}


Accumulation of Senescence-Associated $\beta$-galactosidase (SA- $\beta$-gal) is one of the hallmaker of senescence. EPCs $\left(1 \times 10^{5} / \mathrm{ml}\right)$ were seeded on sterilized coverslips, synchronized growth by FBS-free medium, and incubated by LG, HG for 48h; the NLRP3 inflammasome positive control was induced by LPS treatment for $4 \mathrm{~h}$ and ATP treatment for $1 \mathrm{~h}$. EPCs were washed twice by precooled PBS and fixed by an immobilized reagent in SA- $\beta$-gal kit (C0602, Beyotime, Shanghai, China) for $15 \mathrm{~min}$. Staining solution (Formulated as per kit instructions, $1 \mathrm{ml}$ containing A, B, C and X-Gal solutions $10 \mu \mathrm{l}, 10 \mu \mathrm{l}, 930 \mu \mathrm{l}$, and $50 \mu \mathrm{l}$ ) was added and incubated the cells for $36 \mathrm{~h}$ at $37^{\circ} \mathrm{C}$, avoiding light. The coverslips were moisturized with glycerine PBS (1:1) solution, observed, and photographed by a microscope (Carl Zeiss, Germany, Axio Scope A1) in the light field. The images were processed using ZEN Blue 2.3 software (Carl Zeiss, Germany). Image $\mathrm{J}$ software was used to analyze the percentage of SA- $\beta$-gal positive area (green staining).

\subsection{Statistics}

Statistical analysis was performed with IBM SPSS Statistics 20 software. Data are presented as means \pm SE. Significant differences between and within multiple groups were examined using ANOVA for repeated measures, followed by Duncan's multiple range test. The Independent-Samples t-test was used to detect significant differences between the two groups. $p<0.05$ was considered statistically significant.

\section{Results}

\subsection{STS inhibited EPC NLRP3 inflammasome activation and dysfunction induced by HG}

To observe the activation of the NLRP3 inflammasome by HG on EPC, we used western blot to detect the changes of relevant indicators. We found that the NLRP3 expression and the degree of procaspase-1 converted to caspase- 1 and prolL-1 $\beta$ to IL- $1 \beta$ were increased by HG, which was similar to NLRP3 inflammasome positive control, LPS+ATP treatment (Figure 1A-1B). Further, immunofluorescence results showed that HG and LPS+ATP treatment induced significant merging of NLRP3 (red fluorescence dots) and ASC (green fluorescence dots) to form yellow dots, which represented NLRP3 inflammasome assembly, STS inhibited the assembly (Figure 1E). These changes indicated that HG caused significant activation of NLRP3 inflammasome in EPC. STS inhibited the activation.

Similar to LPS+ATP treatment, HG significantly caused the increase of DNA damage index $y-\mathrm{H} 2 \mathrm{AX}$ expression and positive immunofluorescence in the nucleus (Figure 1C-1D, 1F), cell senescence-related index p21 expression (Figure 1C-1D), and senescence-associate beta-galactosidase (SA- $\beta$-gal) positive area (Figure 1G). At the same time, HG caused the decrease of cell proliferation index Ki67 (Figure 1C-1D) and EPC differentiation function index CD31 and VWF expression on cytomembrane (Figure 1C-1D). STS treatment alleviated the above EPC damage changes caused by both HG and LPS+ATP. 
These results indicated that STS alleviated HG-induced EPC DNA damage, down-regulated proliferation, differentiation, and accelerated senescence while inhibits HG-induced activation of EPC NLRP3 inflammasome.

\subsection{STS ameliorated HG impaired EPC functions by Inhibiting NLRP3 inflammasome activation}

To investigate the possible role of inflammasome activation in HG-induced EPC function impairment, we used NLRP3 inflammasome-specific inhibitor MCC950 to intervene[36]. The results showed that similar to MCC950, STS significantly inhibited the production of inflammasome activation markers active caspase1 and mature IL-1 $\beta$ (Figure 2A-2B), inhibited the expression of $\mathrm{y}-\mathrm{H} 2 \mathrm{AX}$ (Figure 2C-2D) and restored the expression of cell proliferation indexes Ki67 expression (Figure 2C-2D) and BrdU incorporation positive cell area (Figure 2F), EPC differentiation indexes CD31 expression (Figure 2C-2D) and immunofluorescence positive area on the cell membrane (Figure 2E) and the membrane expression of VWF (Figure 2C-2D).

These results indicated that STS improved HG-induced EPC dysfunction by inhibiting the activity of the NLRP3 inflammasome.

\subsection{STS alleviated EPC DNA damage and dysfunction by inhibiting Caspase-1 dependent catalase activity down- regulation}

The above results concluded that inhibition of NLRP3 inflammasome activity might improve HG-induced EPC damage. So, we speculated that caspase-1, the core active product activated by the inflammasome, might play a key role in EPC dysfunction. We used a caspase-1 inhibitor, Z-WEHD-FMK[37], treated EPC, and the results showed that similar to WEHD, STS restored HG damaged CAT activity, and reduced the cleaved CAT production (a fragment with a molecular weight of $34 \mathrm{kDa}$ ) (Figure 3A-3B), inhibited the hydrogen peroxide accumulation (Figure $3 \mathrm{H}$ ); STS decreased the expression of $\mathrm{Y}-\mathrm{H} 2 \mathrm{AX}$ and its positive immunofluorescence area (Figure 3C-3D, 3E); STS reduced the expression of p21, and restored the expression of Ki67, CD31, and VWF (Figure 3C-3D). At the same time, STS and WEHD alleviated EPC damage in NLRP3 inflammasome activation positive control induced by LPS+ATP treatment (Figure 3A3E).

In addition, we used exogenous CAT intervention to observe the EPC function changes. Results showed that exogenous CAT enhanced the protective effect of STS on the HG-induced CAT inactivation, EPC DNA damage, Ki67, CD31 and VWF down-regulation, the p21 up-regulation, and hydrogen peroxide accumulation (Figure 3F-3G, 3I).

These results indicated that NLRP3 inflammasome mediated caspase-1 activation weakened CAT activity and led to EPC DNA damage and dysfunction; STS reversed the damage by protecting CAT activity from caspase-1 activation induced by HG. 


\subsection{STS protected CAT activity by inhibiting NLRP3 inflammasome dependent caspase-1 activation}

To investigate the potential mechanisms of STS protecting HG impaired EPC function, we observed CAT activity following inhibition of NLRP3 inflammasome activation. Results showed that similar to MCC950 treatment STS reduced the active caspase-1 (p20) production, restored the impaired CAT activity, and reduced the Hydrogen peroxide accumulation induced by HG or LPS+ATP (Figure 4A-4B), STS inhibited the HG or LPS+ATP treatment-induced CAT cleavage (Figure 4A-4B). More importantly, we further investigated the possible interaction between caspase-1 and CAT by Co-immunoprecipitation (Co-IP) and immunofluorescence co-localization. Results showed that MCC950 reduced the degree of Co-IP of caspase-1 and CAT (Figure 4C-4D); meanwhile, MCC950 inhibited the formation of yellow spots, which represented co-localization of CAT (red spots) and caspase-1 (green spots) (Figure 4E-4F), STS mimicked the MCC950 effect on inhibiting the interaction between caspase-1 and CAT (Figure 4C-4F). These results indicated that STS inhibited NLRP3 inflammasome dependent active caspase-1 production, and STS protected CAT activity by inhibiting NLRP3 inflammasome dependent caspase-1 activation.

We superficially explored the possible cleavage sites of CAT by caspase-1 using the point mutation method. According to molecular weight estimation and software (Editseq 7.1.0) calculation, we mutated aspartic acid 298, 307, and 226 sites in CAT amino acid sequence into alanine. The point mutant plasmids were transfected into HEK293T cells, which transfected with NLRP3, ASC, and caspase-1 plasmids simultaneously. The NLRP3 inflammasome was activated by LPS+ATP, and then the splice of CAT after the mutant site was observed. The results showed that the D298A mutation prevented CAT splicing following activation of NLRP3 inflammasome (Figure 4G-4H), and D298A mutation also prevented CAT and p20 coprecipitation (Figure 4I-4L). These results indicated that active caspase-1 deactivated CAT by cleaving CAT at D298.

These results indicated that STS protected CAT activity by inhibiting NLRP3 inflammasome-dependent caspase-1 activation and CAT cleavage at the D298 site.

\subsection{STS improved CAT activity and EPC function by inhibiting the RAGE-TXNIP-caspase-1 pathway}

TXNIP is a recognized protein that activates NLRP3 inflammasomes[38]. TXNIP is highly expressed in the $\mathrm{HG}$ environment and causes extensive intracellular oxidative stress and upregulation of inflammatory status. EPCs were treated with TXNIP siRNA for observation. Results showed that TXNIP gene silencing significantly inhibited the activation of caspase-1, CAT activity damage, hydrogen peroxide accumulation, $\mathrm{Y}-\mathrm{H} 2 \mathrm{AX}$, and p21 expression, and restored the expression of Ki67, CD31, and VWF, and STS had a similar effect to TXNIP siRNA (Figure 5A-5C). These results indicated that STS restored CAT activity and EPC proliferation and differentiation functions, inhibited DNA damage and cell senescence of EPC by inhibiting the TXNIP-caspase-1 pathway. 
We further attempted to explore the possible mechanism by which HG impaired EPC function. The content of AGEs in EPC culture supernatant was detected by a commercial AGEs Assay Kit. The results showed that with the increase of HG treated time and concentration, many AGEs were positive in the culture medium (Figure 5D-5E). These results meant that the excess glucose in the culture medium induced AGEs formation. Therefore, we speculated that the AGE-RAGE-TXNIP pathway is likely to play an essential role in impairing EPC function by HG. EPCs were treated with BSA-AGE, and the RAGE pathway was blocked by TTP488, a small-molecule inhibitor of RAGE[39]. The results showed that TTP488 inhibited the TXNIP expression, activation of caspase-1, CAT activity damage, hydrogen peroxide accumulation, $\mathrm{y}-\mathrm{H} 2 \mathrm{AX}$, and $\mathrm{p} 21$ expression, and restore the expression of Ki67, CD31, and STS had a similar effect to TTP488 (Figure 5F-5H). These results indicated that excess glucose was likely to activate the RAGE-TXNIP-caspase-1 pathway through the formation of AGEs analogs to damage CAT activity, resulting in DNA damage, and ultimately impaired the proliferation and differentiation of EPC functions and accelerated EPC senescence.

\subsection{STS enhanced EPC therapy of ameliorating renal function in diabetic mice}

To observe the effect of EPC transplantation on the kidney function in diabetes, we transplanted EPC into diabetic nude mice and observed the changes in kidney functions and the pathological change of glomeruli. The results showed that EPC transplantation did not significantly reduce the blood and urine sugar (Figure 6C-6D), but decreased 24h urine protein level (Figure 6E), the plasma creatinine (Figure 6F), restored the urine creatinine excretion (Figure $6 \mathrm{G}$ ), and suppressed the ratio of urinary albumin/creatinine (Figure 6H) in diabetic mice. STS enhanced the above-improved changes in kidney functions by EPC transplantation (Figure 6E-6H). Pathological examination showed that EPC transplantation reduced glomerular cell loss and amaranth staining (Figure 6A-6B) in STZ treated mice kidneys. STS treatment enhanced the glomerular changes (Figure 6A-6B).

These results suggested that STS prior intravenous infusion enhanced the therapeutic effects of EPC transplantation on glomerular cell well-organizing and glycogen deposition inhibition and kidney function improvement in diabetic mice.

\subsection{STS protected EPC function in diabetic mice glomeruli}

To observe the EPC function changes and the STS protection in diabetic mice glomeruli, CM-Tracker labeled EPCs were transplanted, and immunofluorescence in the frozen glomerular section was detected. Results showed that EPC (red fluorescence) gathered in STZ treated mice glomeruli, but the control glomeruli had very little EPC (Figure 7A-7E); STZ treatment induced TXNIP, p20 and p21 (green immunofluorescence) expression while inhibited Ki67 and CD31 (green immunofluorescence) expression in EPCs those gathered in glomeruli (Figure 7A-7E); STS significantly inhibited the co-localization (yellow staining) of CM-Tracker and TXNIP, p20 and p21 (Figure 7A-7C) while restored the co-localization (yellow staining) of CM-Tracker and Ki67 and CD31 (Figure 7D-7E). 
These results suggested that glomerular vascular endothelium was repaired and integrated by EPC homing, STS protected EPC from TXNIP expression, inflammasome activation, and senescence, restored EPC proliferation and differentiation functions in STZ-induced diabetic pathological environment.

\section{Discussion}

Based on the activation of the NLRP3 inflammasome, this study discussed that the decrease of EPC proliferation and differentiation functions, accelerated senescence induced by HG, the kidney function benefit from EPC transplantation were weakened in diabetic mice, STS protected EPC functions from the diabetic pathological environment. In terms of mechanism, excess glucose activated the RAGE-TXNIPNLRP3 inflammasome pathway through the production of AGEs analogs, leading to caspase-1-mediated CAT inactivation, resulting in the accumulation of hydrogen peroxide, DNA damage, and eventually EPC dysfunction. STS blocked the RAGE-TXNIP-NLRP3 inflammasome pathway to protect the CAT activity, block DNA damage and restore the proliferation and differentiation functions of EPC, slow down its senescence.

\section{The origin and identification typing of EPC remains controversial}

At present, there are some controversies about the source of EPC. For example, some studies proved that EPC does not derive from bone marrow[40]. However, most studies believe that EPC is a bone marrowderived stem cell [41]. Consequently, these controversies indicate that EPC has multiple sources, and this issue needs further research. At present, the typing characteristics of EPC have not been completely determined. Some studies believe that the following functions of the cells are different according to the different antigen markers those emerge on the cell membrane. For example, CD14, CD16, CD34, and VEGFR2 positive cells play the role of angiogenesis, but early EPC also expresses surface markers of bone marrow-derived stem cells such as CD14 and CD45[30]. The most recognized EPC identification includes that cells express positive cell surface markers CD34, CD133, and VEGFR2 (Flk1/KDR); endothelial markers represented by VWF and CD31 are gradually expressed in the later stage; cells can be double labeled with Dil-Ac-LDL and FITC-UEA-l; and cells have the physiological function of repairing damaged vascular endothelium and participating in angiogenesis[32, 42].

Our results showed that compared with mature endothelial cells MAVEC, the isolated and cultured mouse bone marrow-derived cells had all three makers, CD34, CD133, and VEGFR2 positive (supplemental Figure 1B), and were double fluorescence-labeled by Dil-Ac-LDL and FITC-UEA-I (supplemental Figure 1C). The early fusiform growth cell was observed under a light microscope compared with cobble-like colony growth cell and tubule formation ability at the later stage (supplemental Figure 1A). These characteristics were similar to most current studies' conclusions, so the bone marrow-derived cells we isolated and cultured had the recognized EPC characteristics. 


\section{EPC transplantation alleviated the kidney dysfunction in diabetic mice}

The protective effect of EPC on glomerular injury has been reported. In vitro studies, EPC secreted: vascular growth factor, insulin-like growth factor 1, fibroblast growth factor 2, and Hepatocyte growth factor to restore the damage of human glomerular endothelial cells caused by Shigellin[43]. In vitro tissue engineering studies have shown that CD133 positive cells had a potential role in forming artificial glomeruli[44]. EPC transferred encode Factor H, CD55, and CD59 mRNA into mesangial cells through extracellular vesicles inhibited mesangial cell apoptosis induced by anti-Thy 1.1 antibody/complement and C5B-9 /C3 mesangial cell deposition in anti-Thy 1.1 glomerulonephritis[45]. It has been summarized that the endothelial repair mechanism was impaired in the diabetic state, and diabetic patients with proteinuria were accompanied by a decrease in circulating EPC count[46]. In vivo studies have also shown that transplantation of CM-Dil labeled bone marrow-derived stem cells restored the number of glomerular endothelial cells and reduced the area of a-SMA positive regions in the mesangial region and the degree of macrophage infiltration in a rat model of anti-Thy1.1 glomerulonephritis[47]. Conclusions of clinical studies also indicated that EPC had a mitigating effect on DKD[48]; moreover, the impairment of EPC function in the process of chronic renal failure might be associated with inflammation[49]. These findings suggested that EPC transplantation very likely had the potential repairment of the damaged glomerular vascular endothelial cell layer, and the mechanism might be related to EPC differentiate into mature vascular endothelial cells and the EPC paracrine effect. Our conclusion is similar to those in previous studies, EPC transplantation improved glomerular dysfunction. EPC transplantation significantly inhibited proteinuria production, reduced plasma creatinine, increased urinary creatinine excretion and limited the ratio of urinary albumin/creatinine in diabetic mice. More, EPC transplantation provided better organized cell arrangement and less glycogen deposition in diabetic glomerulus. However, the effect of EPC paracrine action on glomerular function has not been involved in this study. Our results focused more on the EPC functions of proliferation and differentiation, as well as the role of cell senescence in DKD, because these results are still lacking.

\section{Caspase-1mediates CAT inactivation}

As the core product of the assembly of the NLRP3 inflammasome, caspase- 1 has the specific aspartic acid cysteine proteinase activity and the most widely known role in mediating the maturation of IL-1 $\beta$ and IL-18, and the activation of GSDMD, which plays the crucial role in pyroptosis[50]. Furthermore, according to studies, there are more than 1000 substrates of caspase-1, those are affected cytoskeleton structure, programmed cell death, glucose, and lipid metabolism, autophagy flow, and even cell differentiation and other cellular functions [51-55]. The results presented in this study showed that caspase-1 might interact with CAT. The active caspase- 1 cleaved CAT to produce a fragment of about $34 \mathrm{kDa}$, and then CAT enzyme activity decreased, which further led to hydrogen peroxide accumulation, DNA damage, and eventually EPC dysfunction. Previous findings seemed to provide some potential correlation between the 
NLRP3 inflammasome-caspase-1 pathway and CAT activity. CAT activity tends to decrease with activation of NLRP3 inflammasomes[56-58],but inhibition of NLRP3 inflammasome played a role in restoring CAT activity[59]. However, it is still unclear whether the reason for the decrease in CAT activity is related to the activity of caspase-1. A study showed that CAT might be one of the substrates of caspase1[55] खbut there is no specific study on it at present. Our results showed that caspase-1 significantly interacted with CAT and might cleave it at the site of aspartic acid 298. This result partially verified the speculation of previous studies that CAT was one of the substrates of caspase- 1 . Previous studies have also concluded that antioxidant superoxide dismutase and CAT inhibited homocysteine/lipopolysaccharide-induced caspase- 1 activation[60, 61]. This conclusion showed that CAT inhibited the activation of caspase-1, which was different from our findings that caspase-1 inactivated CAT activity. The reason may be that reactive oxygen species are recognized as inducers of the activation of NLRP3 inflammasomes[62]. Our results suggested that CAT inactivation caused by activated caspase-1 further aggravated the accumulation of reactive oxygen species, worsened the NLRP3 inflammasome activation environment, and formed a progressively aggravating inflammasome activation loop. We did find that exogenous CAT-PEG treatment contributed to the improvement of the damaged EPC function, but whether it was related to the mitigation of NLRP3 inflammasome mediated caspase-1 activation remains to be further investigated.

\section{HG-induced EPC dysfunction by activating TXNIP-NLRP3 inflammasome pathway through sthe formation of AGEs}

Treatment with HG and NLRP3 inflammasome positive control LPS+ATP, EPC had similar effects on activating NLRP3 inflammasome and impairing cellular function, while treatment with MCC950, a specific inhibitor of NLRP3, inhibited the impairment of EPC function. This result indicated that HG activated the NLRP3 inflammasome and played a role in impairing EPC function. However, there is no unified theoretical model for the mechanism of HG regulating the activation of NLRP3 inflammasome. Some studies suggested that HG activated NLRP3 inflammasome by activating TLR4/NFKB and ROS/TXNIP pathway[63-65],but exactly how HG interacted with TLR4 receptors is still unclear. Recent studies have found that RAGE and TLR4 activated respiratory inflammatory response through sharing HMGB1 as a ligand to form RAGE/HMGB1 signaling pathway[66]; meanwhile, excess glucose in the HG medium reacted with protein in serum to generate AGE, which caused inflammatory diabetic cardiomyopathy[67]. These conclusions indicated that the HG-AGE-RAGE pathway might play a vital role in the activation of NLRP3 inflammasomes. Our results preliminarily supported this inference. We detected the AGE analogs in the HG medium, and AGEs were significantly higher than those in the LG medium when EPC was incubated at $37^{\circ} \mathrm{C}$ for up to 72 hours. We also used Azeliragon (TTP488), a specific inhibitor of RAGE, which blocked the activation of NLRP3 inflammasome induced by HG. TTP488 inhibited the activation of NLRP3 inflammasomes and EPC dysfunctions. These results suggested that excessive glucose in the EPC medium partially formed AGE analogs, which activated RAGE and led to activation of the NLRP3 inflammasome. Studies concluded that TXNIP interacted with the NLRP3 
molecule and caused the activation of the NLRP3 inflammasome[38]. Our results showed that genesilencing TXNIP inhibited the activation of NLRP3 inflammasome and HG-induced impairment of EPC function. This conclusion is similar to those in previous studies that inhibition of TXNIP expression synchronized inhibition of NLRP3 inflammasome in EPC and gene silence or knockout of TXNIP eliminated HG-induced NLRP3 inflammasome activation in islet beta cells[68-70]. We found that inhibition of HG-induced TXNIP expression down-regulated the activity of NLRP3 inflammasome and improved EPC functions. Our findings were consistent with previous studies. HG and AGEs upregulated the TXNIP expression [71, 72], and the RAGE-TXNIP pathway has also been shown to play a crucial role in a variety of cellular functional impairments [73-75]. Therefore, our results concluded that HG activated the RAGE-TXNIP-NLRP3 inflammasome pathway through the formation of AGEs. The pathway was firstly observed in EPC and revealed one of the underlying mechanisms of EPC dysfunction in diabetes.

\section{STS protected EPC function in diabetic pathology}

A few studies have shown that tanshinone $\triangle A$ has a protective effect on EPC function impairment, and the mechanism was related to the inhibition of the production and release of inflammatory factors associated with TNF-a-induced activation of NFKB signal. For example, Tanshinone $\triangle A$ reversed TNF-adamaged proliferation, migration, adhesion, and angiogenesis function of EPC, and inhibited EPC secretion of inflammatory cytokines[27], also, inhibition of NF-KB activation and phosphorylation of IкB-a down-regulated EPC vascular cell adhesion molecule-1 (VCAM)/intracellular adhesion molecule-1 (ICAM-

1) expression, those play a key role in the recruitment of inflammatory cell infiltration in early atherosclerotic lesions[28]. Our results showed a similar conclusion that STS inhibited NLRP3 inflammasome, improved EPC proliferation, and differentiation, and slowed down its senescence. Our results suggested that STS inhibited the activity of NLRP3 inflammasome by inhibiting TXNIP expression, which is similar to the conclusion that STS protects against ischemia/reperfusion myocardial injury[76]. Our findings demonstrated for the first time that STS protected EPC function by inhibiting the RAGETXNIP-NLRP3 inflammasome pathway and improved diabetic glomerulopathy, enriched the protective effect of STS on EPC function, and confirmed its mechanism related to inflammation. It has been reported that STS enhanced CAT activity and protein level of CAT in Human Neuroblastoma Cells[77]; HG inhibited CAT expression and induced oxidative stress in EPC[29]. But the CAT impairing mechanism is poorly studied now. Our results suggested that HG promoted CAT cleavage and inactivation by forming AGEs to activate the RAGE-TXNIP-NLRP3 inflammasome-caspase-1 pathway, which inhibited by STS to protect CAT activity. The renal protective effects of STS have been reported. Tanshinone IIA blocked renal fibrogenesis and inflammation by inhibiting the GSK3 $\beta$ activation in folic acid-induced acute kidney injury, and the following progressive chronic kidney disease [78], and also Tanshinone IIA inhibited the thickening of glomerular basement membrane and the collagen deposition by attenuating PERK signaling activities to reduce endoplasmic reticulum stress in STZ-induced diabetic nephropathy[23]. But the protective effect of STS on glomerular capillary is lack of study. Our results suggested that STS protected the glomerular vascular endothelium repairment of EPC and enhanced the renal function recovery by EPC transplantation in STZ induced diabetic mice. However, our conclusion about glomerular 
vascular endothelium repairment of EPC was inferred mainly based on the recovery of CD31 membrane expression after EPC transplantation and STS injection, the glomerular vascular endothelium repairment of EPC needs further study in a more microscopic way. The VWF and CD31 membrane expression was insufficiently studied in EPC differentiating into mature endothelial cells, and the mechanism of VWF and CD31 coming to the surface of cytomembrane also needs to explore in further study.

\section{Conclusions}

Our results conclude that under diabetic conditions, $\mathrm{HG}$ promoted CAT cleavage and inactivation through activation of the RAGE-TXNIP-NLRP3 inflammasome-caspase-1 pathway, resulting in the accumulation of hydrogen peroxide and DNA damage and EPC function impairment. STS protected CAT activity and EPC function from RAGE-TXNIP-NLRP3 inflammasome pathway activation. EPC transplantation effectively restored the renal function in diabetic mice, which was enhanced by STS treatment. Our conclusion provided a new sight of stem cell therapy in diabetic kidney disease.

\section{Abbreviations}

GFB: glomerular filtration barrier; GEC: Glomerular endothelial cell ; DKD: Diabetic Kidney Disease; ESRD: End-Stage Renal Disease; EPC: Endothelial progenitor cell; EC: Endothelial Cell; VSMC: vascular smooth muscle cell; STS: Sodium Tanshinone Sulfonate $\triangle A$; HG: High Glucose; TXNIP: Thioredoxin interacting Protein; NLRP3 inflammasome: NLR family pyrin domain containing 3 inflammasome; CAT: catalase; AGEs: Advanced Glycation End Products RAGE: Receptor of AGE; STZ: Streptozotocin; FBG: fasting blood glucose; SA- $\beta$-gal: Senescence-Associated $\beta$-galactosidase.

\section{Declarations}

\section{Consent for publication}

Not applicable.

\section{Availability of data and materials}

The datasets used and analyzed in current study are available from the corresponding author based on reasonable request

\section{Author contributions:}

HYY and WW: laboratory work, data analysis and interpretation, manuscript writing, and financial support; WW: conception and study design and final approval of the manuscript; ZXY, WFF, ZPF: manuscript proofreading and administrative support. All authors have read and approved the manuscript. 


\section{Acknowledgements}

Not applicable.

\section{Funding}

This work was supported by grants from the Youth Project of Heping Hospital Affiliated to Changzhi Medical College (HPYJ201921); Supported by Scientific and Technological Innovation Programs (STIP) of Higher Education Institutions in Shanxi.

\section{Competing interests}

The authors declared no potential conflicts of interest.

\section{Ethics approval and consent to participate}

Animal handling and experimental procedures were approved by the ethics committee of Changzhi medical college (DW2020045), in accordance with the Guidelines of Animal Experiment set by the Bureau of Sciences and Techniques of Shanxi Province, China.

\section{References}

1. Gnudi L, Coward RJM, Long DA. Diabetic Nephropathy: Perspective on Novel Molecular Mechanisms. Trends in endocrinology metabolism: TEM. 2016;27:820-30.

2. Parvanova Al, Trevisan R, lliev IP, et al. Insulin resistance and microalbuminuria: a cross-sectional, case-control study of 158 patients with type 2 diabetes and different degrees of urinary albumin excretion. Diabetes. 2006;55:1456-62.

3. Lenoir $\mathrm{O}$, Jasiek M, Hénique $\mathrm{C}$, et al. Endothelial cell and podocyte autophagy synergistically protect from diabetes-induced glomerulosclerosis. Autophagy. 2015;11:1130-45.

4. Zhang Y, Ma KL, Gong YX, et al. Platelet Microparticles Mediate Glomerular Endothelial Injury in Early Diabetic Nephropathy. Journal of the American Society of Nephrology: JASN. 2018;29:2671-95.

5. Satchell SC, Braet F. Glomerular endothelial cell fenestrations: an integral component of the glomerular filtration barrier. American journal of physiology Renal physiology. 2009;296:F947-56.

6. Sörensson J, Björnson A, Ohlson M, et al. Synthesis of sulfated proteoglycans by bovine glomerular endothelial cells in culture. American journal of physiology Renal physiology. 2003;284:F373-80.

7. Fu J, Wei C, Zhang W, et al. Gene expression profiles of glomerular endothelial cells support their role in the glomerulopathy of diabetic mice. Kidney international. 2018;94:326-45. 
8. Xie RY, Fang XL, Zheng XB, et al. Salidroside and FG-4592 ameliorate high glucose-induced glomerular endothelial cells injury via HIF upregulation. Biomedicine pharmacotherapy = Biomedecine pharmacotherapie. 2019;118:109175.

9. Kirton JP, Xu Q. Endothelial precursors in vascular repair. Microvascular research. 2010;79:193-9.

10. Yu CG, Zhang N, Yuan SS, et al. Endothelial Progenitor Cells in Diabetic Microvascular Complications: Friends or Foes? Stem cells international. 2016;2016:1803989.

11. Pyšná A, Bém R, Němcová $A$, et al. Endothelial Progenitor Cells Biology in Diabetes Mellitus and Peripheral Arterial Disease and their Therapeutic Potential. 2019;15:157-165.

12. Leone $A M$, Valgimigli $M$, Giannico $M B$, et al. From bone marrow to the arterial wall: the ongoing tale of endothelial progenitor cells. European heart journal. 2009;30:890-9.

13. Psaltis PJ, Simari RD. Vascular wall progenitor cells in health and disease. Circulation research. 2015;116:1392-412.

14. Kang H, Ma X, Liu J, et al. High glucose-induced endothelial progenitor cell dysfunction. Diabetes vascular disease research. 2017;14:381-94.

15. Sadanandan N, Di Santo S, Widmer HR. Another win for endothelial progenitor cells: Endothelial progenitor cell-derived conditioned medium promotes proliferation and exerts neuroprotection in cultured neuronal progenitor cells. Brain circulation. 2019;5:106-11.

16. Mudyanadzo TA. Endothelial Progenitor Cells and Cardiovascular Correlates. Cureus. 2018;10:e3342.

17. Wang RY, Liu LH, Liu H, et al. Nrf2 protects against diabetic dysfunction of endothelial progenitor cells via regulating cell senescence. International journal of molecular medicine. 2018;42:1327-40.

18. Fadini GP, Boscaro E, de Kreutzenberg S, et al. Time course and mechanisms of circulating progenitor cell reduction in the natural history of type 2 diabetes. Diabetes care. 2010;33:1097-102.

19. Gao S, Liu Z, Li H, et al. Cardiovascular actions and therapeutic potential of tanshinone IIA. Atherosclerosis. 2012;220:3-10.

20. Ansari MA, Khan FB, Safdari HA, et al. Prospective therapeutic potential of Tanshinone IIA: An updated overview. Pharmacological research. 2021;164:105364.

21. Han D, Wu X, Liu L, et al. Sodium tanshinone IIA sulfonate protects ARPE-19 cells against oxidative stress by inhibiting autophagy and apoptosis. Scientific reports. 2018;8:15137.

22. Liu Y, Tong $C$, Tang $Y$, et al. Tanshinone IIA alleviates blast-induced inflammation, oxidative stress and apoptosis in mice partly by inhibiting the $\mathrm{PI3K} / \mathrm{Akt} /$ FoxO1 signaling pathway. Free radical biology medicine. 2020;152:52-60.

23. Xu S, He L, Ding K, et al. Tanshinone IIA Ameliorates Streptozotocin-Induced Diabetic Nephropathy, Partly by Attenuating PERK Pathway-Induced Fibrosis. Drug design, development and therapy. 2020;14:5773-5782.

24. Li W, Sargsyan D, Wu R, et al. DNA Methylome and Transcriptome Alterations in High GlucoseInduced Diabetic Nephropathy Cellular Model and Identification of Novel Targets for Treatment by Tanshinone IIA. 2019;32:1977-1988. 
25. Zhu J, Xu Y, Ren G, et al. Tanshinone IIA Sodium sulfonate regulates antioxidant system, inflammation, and endothelial dysfunction in atherosclerosis by downregulation of CLIC1. European journal of pharmacology. 2017;815:427-36.

26. Cui ZT, Liu JP, Wei WL. The effects of tanshinone IIA on hypoxia/reoxygenation-induced myocardial microvascular endothelial cell apoptosis in rats via the JAK2/STAT3 signaling pathway. Biomedicine \& pharmacotherapy $=$ Biomedecine \& pharmacotherapie. 2016;83:1116-1126.

27. Wang XX, Yang JX, Pan YY, et al. Protective effects of tanshinone âtuA on endothelial progenitor cells injured by tumor necrosis factor-a. Molecular medicine reports. 2015;12:4055-62.

28. Yang JX, Pan YY, Ge JH, et al. Tanshinone II A Attenuates TNF-a-Induced Expression of VCAM-1 and ICAM-1 in Endothelial Progenitor Cells by Blocking Activation of NF-KB. Cellular physiology and biochemistry: international journal of experimental cellular physiology, biochemistry, and pharmacology. 2016;40:195-206.

29. Dai X, Yan X, Zeng J, et al. Elevating CXCR7 Improves Angiogenic Function of EPCs via Akt/GSK3ß/Fyn-Mediated Nrf2 Activation in Diabetic Limb Ischemia. Circulation research. 2017;120:e7-23.

30. Li Calzi S, Neu MB, Shaw LC, et al. Endothelial progenitor dysfunction in the pathogenesis of diabetic retinopathy: treatment concept to correct diabetes-associated deficits. The EPMA journal. 2010;1:88100.

31. Hristov M, Erl W, Weber PC. Endothelial progenitor cells: mobilization, differentiation, and homing. Arteriosclerosis, thrombosis, and vascular biology. 2003;23:1185-1189.

32. Hristov M, Erl W, Weber PC. Endothelial progenitor cells: isolation and characterization. Trends in cardiovascular medicine. 2003;13:201-6.

33. Peng S, Wei P, Lu Q, et al. Beneficial Effects of Poplar Buds on Hyperglycemia, Dyslipidemia, Oxidative Stress, and Inflammation in Streptozotocin-Induced Type-2 Diabetes. Journal of Immunology Research.

34. Wei W, Li XX, Xu M. Inhibition of vascular neointima hyperplasia by FGF21 associated with FGFR1/Syk/NLRP3 inflammasome pathway in diabetic mice. Atherosclerosis. 2019;289:132-42.

35. Zintel S, Bernhardt D, Rogowska-Wrzesinska A, et al. PaCATB, a secreted catalase protecting Podospora anserina against exogenous oxidative stress. Aging. 2011;3:768-81.

36. Coll RC, Hill JR. MCC950 directly targets the NLRP3 ATP-hydrolysis motif for inflammasome inhibition. 2019;15:556-559.

37. Hussain S, Sangtian S, Anderson SM, et al. Inflammasome activation in airway epithelial cells after multi-walled carbon nanotube exposure mediates a profibrotic response in lung fibroblasts. Particle fibre toxicology. 2014;11:28.

38. Elliott El, Sutterwala FS. Initiation and perpetuation of NLRP3 inflammasome activation and assembly. Immunological reviews. 2015;265:35-52.

39. Davis HM, Essex AL, Valdez S, et al. Short-term pharmacologic RAGE inhibition differentially affects bone and skeletal muscle in middle-aged mice. Bone. 2019;124:89-102. 
40. Fujisawa T, Tura-Ceide O, Hunter A, et al. Endothelial Progenitor Cells Do Not Originate From the Bone Marrow. Circulation. 2019;140:1524-6.

41. Khakoo AY, Finkel T. Endothelial progenitor cells. Annual review of medicine. 2005;56:79-101.

42. Avouac J, Uzan G, Kahan A, et al. Endothelial progenitor cells and rheumatic disorders. Joint bone spine. 2008;75:131-7.

43. Patry C, Betzen C, Fathalizadeh F, et al. Endothelial progenitor cells accelerate endothelial regeneration in an in vitro model of Shigatoxin-2a-induced injury via soluble growth factors. American journal of physiology. Renal physiology. 2018;315:F861-f869.

44. Vu DM, Masuda H, Yokoyama TA, et al. CD133+ endothelial progenitor cells as a potential cell source for a bioartificial glomerulus. Tissue engineering Part A. 2009;15:3173-82.

45. Cantaluppi V, Medica D, Mannari C, et al. Endothelial progenitor cell-derived extracellular vesicles protect from complement-mediated mesangial injury in experimental anti-Thy 1.1 glomerulonephritis. Nephrology, dialysis, transplantation: official publication of the European Dialysis and Transplant Association -. European Renal Association. 2015;30:410-22.

46. Eleftheriadis T, Antoniadi G, Pissas G, et al. The renal endothelium in diabetic nephropathy. Renal failure. 2013;35:592-9.

47. Uchimura H, Marumo T, Takase 0 , et al. Intrarenal injection of bone marrow-derived angiogenic cells reduces endothelial injury and mesangial cell activation in experimental glomerulonephritis. Journal of the American Society of Nephrology: JASN. 2005;16:997-1004.

48. Awal HB, Nandula SR, Domingues CC, et al. Linagliptin, when compared to placebo, improves CD34+ve endothelial progenitor cells in type 2 diabetes subjects with chronic kidney disease taking metformin and/or insulin: a randomized controlled trial. 2020;19:72.

49. Krieter DH, Fischer R, Merget K, et al. Endothelial progenitor cells in patients on extracorporeal maintenance dialysis therapy. Nephrology, dialysis, transplantation: official publication of the European Dialysis and Transplant Association -. European Renal Association. 2010;25:4023-31.

50. Swanson KV, Deng M. The NLRP3 inflammasome: molecular activation and regulation to therapeutics. 2019;19:477-489.

51. Shao W, Yeretssian G, Doiron K, et al. The caspase-1 digestome identifies the glycolysis pathway as a target during infection and septic shock. The Journal of biological chemistry. 2007;282:36321-9.

52. Lamkanfi M, Kanneganti TD, Van Damme P, et al. Targeted peptidecentric proteomics reveals caspase-7 as a substrate of the caspase-1 inflammasomes. Molecular cellular proteomics: MCP. 2008;7:2350-63.

53. Jamilloux Y, Lagrange B, Di Micco A, et al. A proximity-dependent biotinylation (BiolD) approach flags the p62/sequestosome-1 protein as a caspase-1 substrate. 2018;293:12563-12575.

54. Niu Z, Shi Q, Zhang W, et al. Caspase-1 cleaves PPARy for potentiating the pro-tumor action of TAMs. Nature communications. 2017;8:766. 
55. Denes A, Lopez-Castejon G, Brough D. Caspase-1: is IL-1 just the tip of the ICEberg? Cell death disease. 2012;3:e338.

56. Chen Y, Que R, Lin L, et al. Inhibition of oxidative stress and NLRP3 inflammasome by Saikosaponind alleviates acute liver injury in carbon tetrachloride-induced hepatitis in mice. 2020;34:2058738420950593.

57. Sun W, Zeng C, Yue D, et al. Ageratina adenophora causes spleen toxicity by inducing oxidative stress and pyroptosis in mice. 2019;6:190127.

58. Wei J, Wang X, Liu Q, et al. The impact of polystyrene microplastics on cardiomyocytes pyroptosis through NLRP3/Caspase-1 signaling pathway and oxidative stress in Wistar rats. 2021;36:935-944.

59. Dwivedi DK, Jena GB. NLRP3 inhibitor glibenclamide attenuates high-fat diet and streptozotocininduced non-alcoholic fatty liver disease in rat: studies on oxidative stress, inflammation, DNA damage and insulin signalling pathway. 2020;393:705-716.

60. Xi H, Zhang Y, Xu Y, et al. Caspase-1 Inflammasome Activation Mediates Homocysteine-Induced Pyrop-Apoptosis in Endothelial Cells. Circulation research. 2016;118:1525-39.

61. Abais JM, Xia M, Li G, et al. Contribution of endogenously produced reactive oxygen species to the activation of podocyte NLRP3 inflammasomes in hyperhomocysteinemia. Free radical biology medicine. 2014;67:211-20.

62. Zhou R, Yazdi AS, Menu P, et al. A role for mitochondria in NLRP3 inflammasome activation. Nature. 2011;469:221-5.

63. Leng B, Zhang Y, Liu X, et al. Astragaloside IV Suppresses High Glucose-Induced NLRP3 Inflammasome Activation by Inhibiting TLR4/NF-KB and CaSR. 2019;2019:1082497.

64. Lu L, Lu Q. Vitamin D(3) Protects against Diabetic Retinopathy by Inhibiting High-Glucose-Induced Activation of the ROS/TXNIP/NLRP3 Inflammasome Pathway. 2018;2018:8193523.

65. Moraes-Vieira PM, Yore MM, Sontheimer-Phelps A, et al. Retinol binding protein 4 primes the NLRP3 inflammasome by signaling through Toll-like receptors 2 and 4. 2020;117:31309-31318.

66. Ullah MA, Loh Z, Gan WJ, et al. Receptor for advanced glycation end products and its ligand highmobility group box-1 mediate allergic airway sensitization and airway inflammation. The Journal of allergy clinical immunology. 2014;134:440-50.

67. Wang Y, Luo W, Han J, et al. MD2 activation by direct AGE interaction drives inflammatory diabetic cardiomyopathy. 2020;11:2148.

68. Deng Y, Han X, Yao Z, et al. PPARa Agonist Stimulated Angiogenesis by Improving Endothelial Precursor Cell Function Via a NLRP3 Inflammasome Pathway. Cellular physiology and biochemistry: international journal of experimental cellular physiology, biochemistry, and pharmacology. 2017;42:2255-2266.

69. Zhou R, Tardivel A, Thorens B, et al. Thioredoxin-interacting protein links oxidative stress to inflammasome activation. Nature immunology. 2010;11:136-40. 
70. Oslowski CM, Hara T, O'Sullivan-Murphy B, et al. Thioredoxin-interacting protein mediates ER stressinduced $\beta$ cell death through initiation of the inflammasome. Cell metabolism. 2012;16:265-73.

71. Devi TS, Somayajulu M, Kowluru RA, et al. TXNIP regulates mitophagy in retinal Müller cells under high-glucose conditions: implications for diabetic retinopathy. Cell death disease. 2017;8:e2777.

72. Kong X, Lu AL, Yao XM, et al. Activation of NLRP3 Inflammasome by Advanced Glycation End Products Promotes Pancreatic Islet Damage. 2017;2017:9692546.

73. Sbai O, Devi TS, Melone MA, et al. RAGE-TXNIP axis is required for S100B-promoted Schwann cell migration, fibronectin expression and cytokine secretion. Journal of cell science. 2010;123:4332-9.

74. Jiang $M$, Wang $X$, Wang $P$, et al. Inhibitor of RAGE and glucose-induced inflammation in bone marrow mesenchymal stem cells: Effect and mechanism of action. Molecular medicine reports. 2020;22:3255-62.

75. Perrone L, Devi TS, Hosoya K, et al. Thioredoxin interacting protein (TXNIP) induces inflammation through chromatin modification in retinal capillary endothelial cells under diabetic conditions. Journal of cellular physiology. 2009;221:262-72.

76. Hu Q, Wei B, Wei L, et al. Sodium tanshinone IIA sulfonate ameliorates ischemia-induced myocardial inflammation and lipid accumulation in Beagle dogs through NLRP3 inflammasome. International journal of cardiology. 2015;196:183-92.

77. Li H, Han W, Wang H, et al. Tanshinone IIA Inhibits Glutamate-Induced Oxidative Toxicity through Prevention of Mitochondrial Dysfunction and Suppression of MAPK Activation in SH-SY5Y Human Neuroblastoma Cells. 2017;2017:4517486.

78. Jiang C, Zhu W, Yan X, et al. Rescue therapy with Tanshinone IIA hinders transition of acute kidney injury to chronic kidney disease via targeting GSK3 $\beta$. Scientific reports. 2016;6:36698.

\section{Figures}



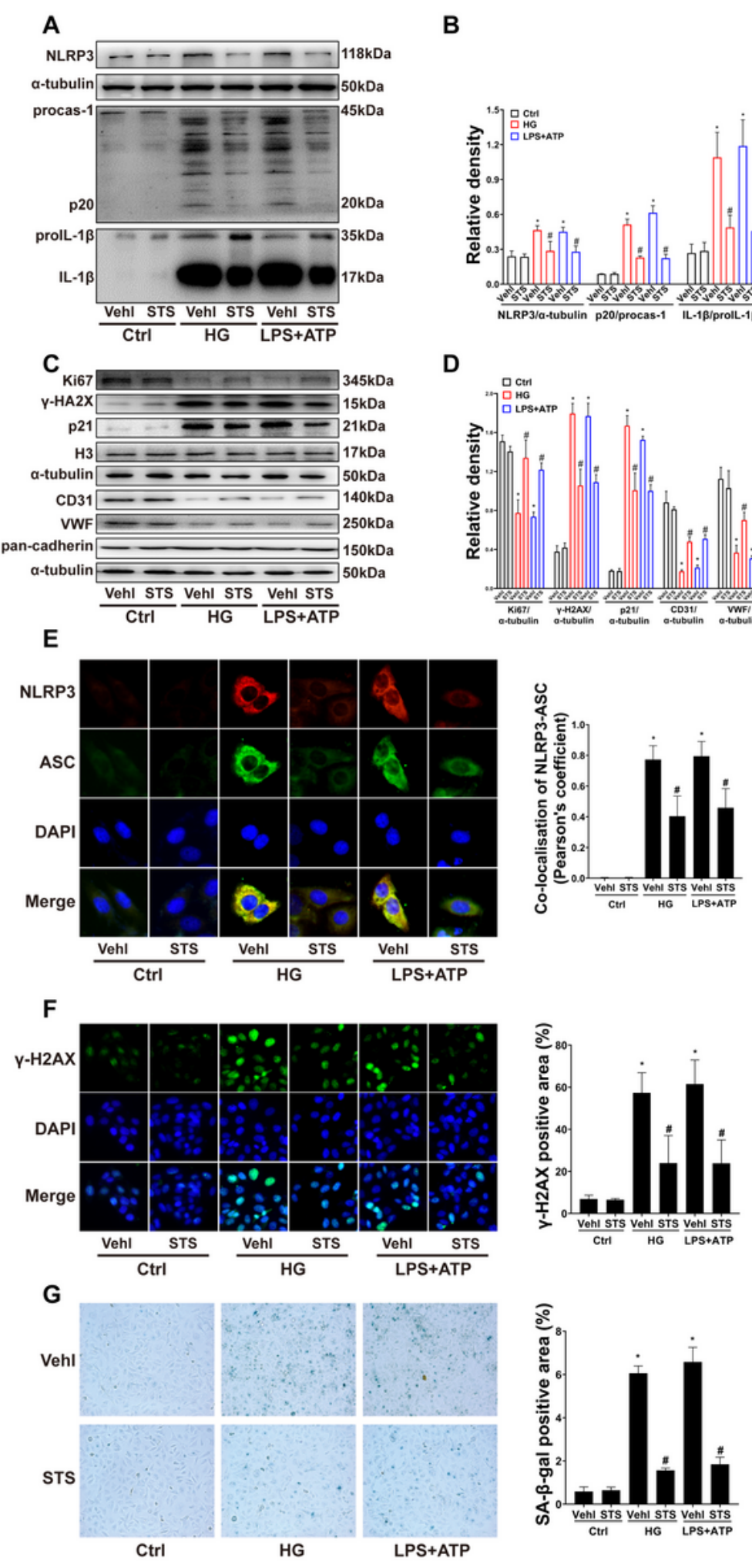

\section{Figure 1}

STS inhibited EPC NLRP3 inflammasome activation and dysfunction induced by HG (A-B):

Representative Western blot gel and summarized data show the protein expression of NLRP3, p20, and IL$1 \beta$ production; (C-D): Representative Western blot gel and summarized data show the protein of Ki67, $\gamma^{-}$ $\mathrm{H} 2 \mathrm{AX}, \mathrm{p} 21$ expression in the nucleus, CD31, VWF expression on the cell cytomembrane, $\mathrm{H} 3$ and pancadherin as the normalization of nucleus protein and cytomembrane protein respectively (the full-length 
gels were showed in supplemental figure 2-A); (E): Representative immunofluorescence images (200x) and summarized coefficient of co-localization of NLRP3 and ASC; (F): Representative immunofluorescence images of $\mathrm{Y}-\mathrm{H} 2 \mathrm{AX}$ in the nucleus $(100 x)$ and summarized percent of the $\mathrm{Y}-\mathrm{H} 2 \mathrm{AX}$ positive area in nucleus total area; (G): Representative images of SA- $\beta$-gal staining (50x) and summarized data show the percent of SA- $\beta$-gel positive area. ${ }^{*} p<0.05$ vs. Control (Ctrl); $\# p<0.05$ vs. HG or LPS+ATP treated group $(n=3)$.

A

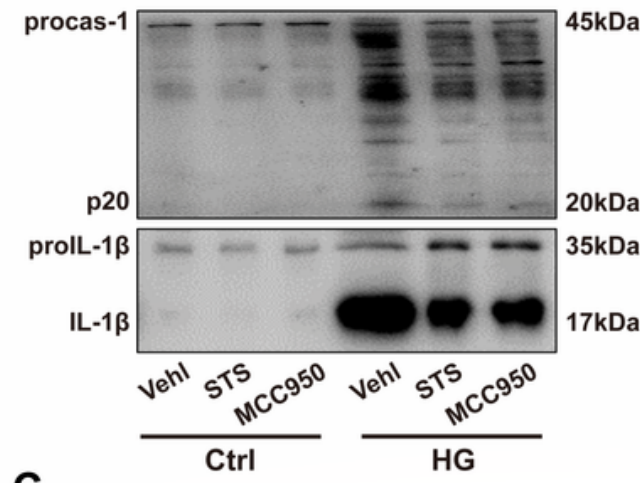

C
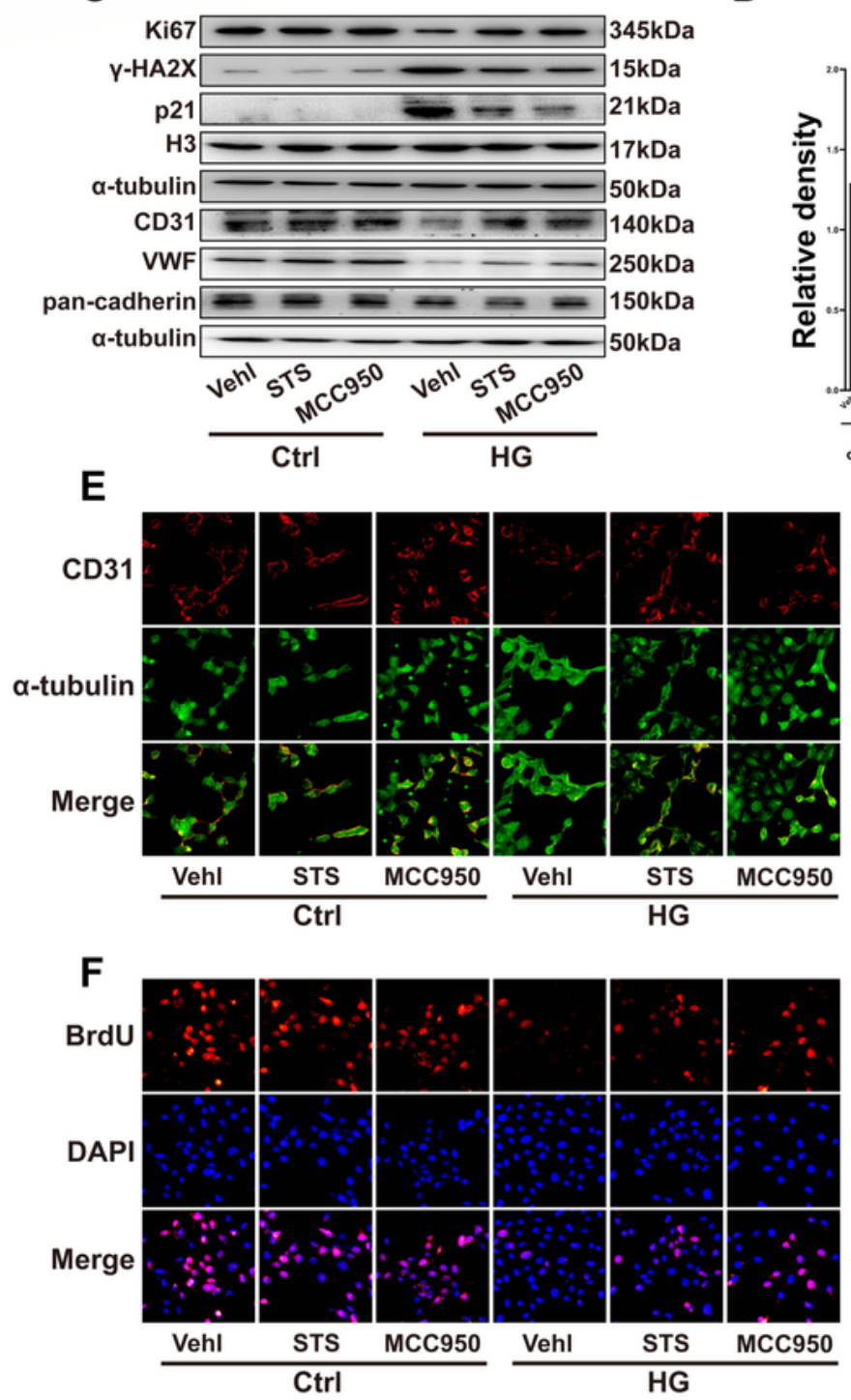

\section{B}
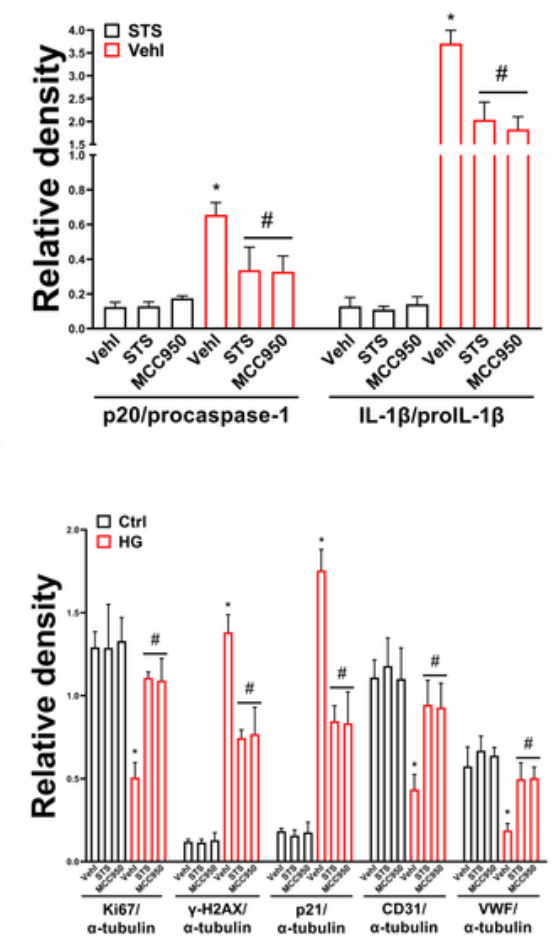

Figure 2
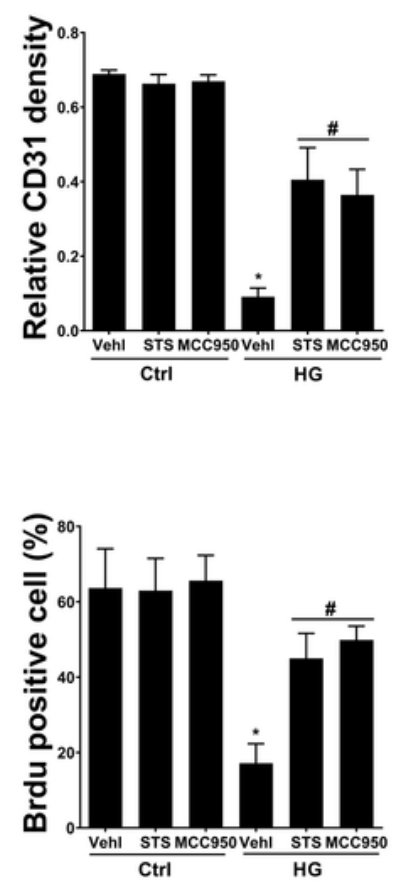
STS ameliorated HG impaired EPC functions by inhibiting NLRP3 inflammasome activation (A-B): Representative Western blot gel and summarized data show the $\mathrm{p} 20$ and IL-1 $\beta$ production; (C-D): Representative Western blot gel and summarized data show the protein of Ki67, $\mathrm{Y}-\mathrm{H} 2 \mathrm{AX}$, p21 expression in the nucleus, CD31, VWF expression on the cell cytomembrane, $\mathrm{H} 3$ and pan-cadherin as the normalization of nucleus protein and cytomembrane protein respectively (the full-length gels were showed in supplemental figure 2-B); (E): Representative images of CD31 on cell cytomembrane (100x), atubulin as control, and summarized relative CD31 density; (F): Representative immunofluorescence images of BrdU incorporation (100x) and summarized percent of BrdU positive area in DAPI. * $p<0.05$ vs. Control (Ctrl); \#p<0.05 vs. HG treated group $(\mathrm{n}=3)$. 
A

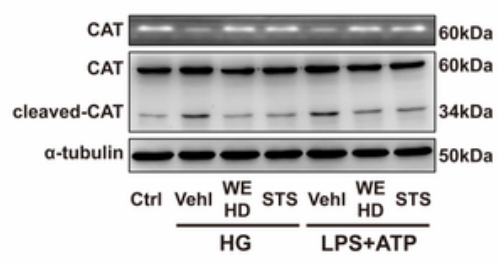

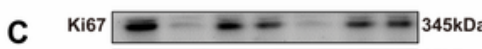
$\mathrm{Y}$-HA2X $-\ldots-\ldots-\ldots$

p21 $-------21 \mathrm{kDa}$

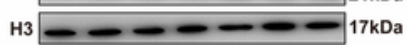
$\alpha$-tubulin $--\longrightarrow-\square$ - $50 \mathrm{kDa}$

CD31 $-\quad-\ldots-140 \mathrm{kDa}$

vWF $-\ldots 250 \mathrm{kDa}$ pan-cadherin $\rightarrow-m+n-m$ 150ka

$\alpha$-tubulin $-\ldots$ - $-\infty$ 50kDa Ctrl Vehl ${ }_{\text {HD }}^{\text {WE }}$ STS Vehl ${ }_{\text {HD }}^{\text {WE }}$ STS

E

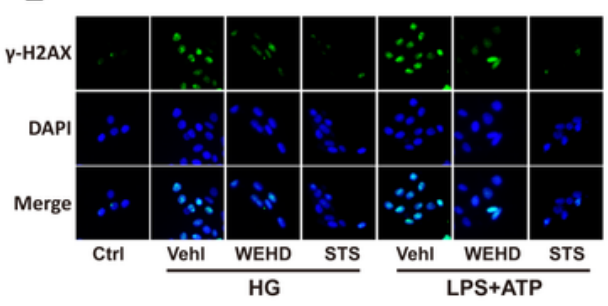

$\mathbf{F}$

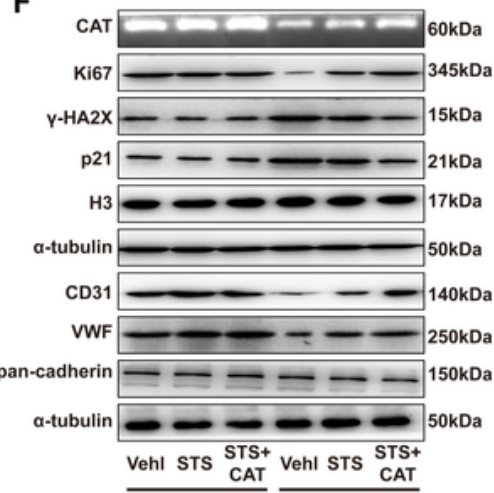

$\mathrm{H}$

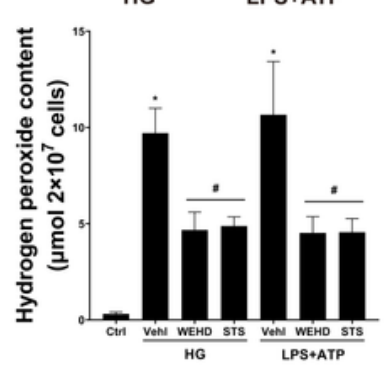

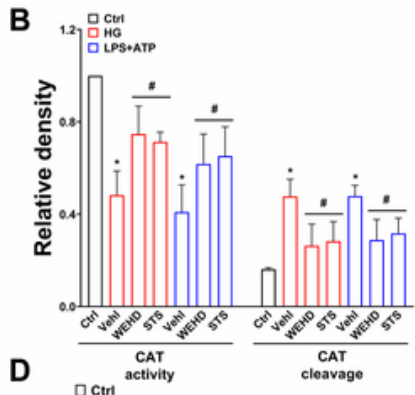
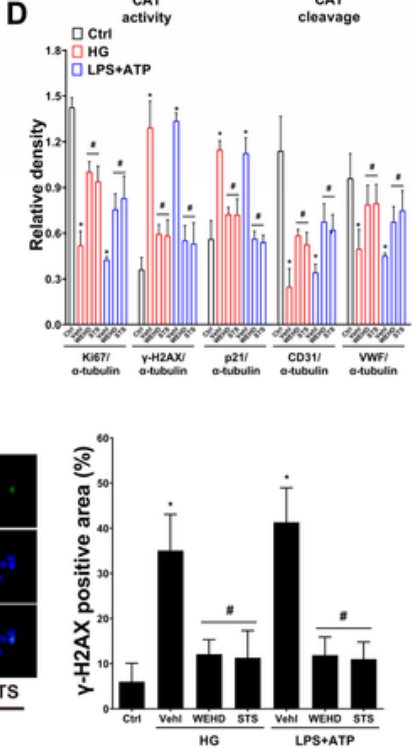

G
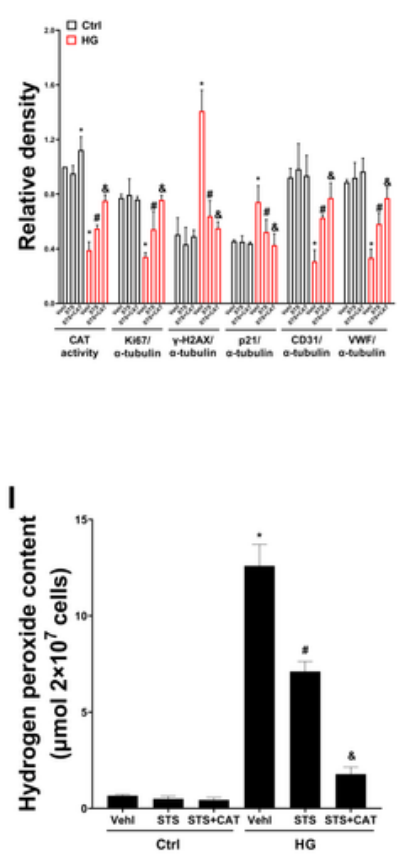

Figure 3

STS alleviated EPC DNA damage and dysfunction by inhibiting Caspase-1 dependent catalase activity down-regulation (A-B): Representative PAGE gel and summarized data show the CAT activity, representative Western blot gel and summarized data show and the degree of CAT cleavage; (C-D): Representative Western blot gel and summarized data show the protein of Ki67, $\mathrm{Y}-\mathrm{H} 2 \mathrm{AX}$, p21 expression in the nucleus, CD31, VWF expression on the cell cytomembrane, $\mathrm{H} 3$ and pan-cadherin as the 
normalization of nucleus protein and cytomembrane protein respectively (the full-length gels were showed in supplemental figure 2-C); (E): Representative immunofluorescence images of $\mathrm{Y}-\mathrm{H} 2 \mathrm{AX}$ in the nucleus (100x) and summarized data show percent of the $\mathrm{Y}-\mathrm{H} 2 \mathrm{AX}$ positive area in nucleus total area; ( $\mathrm{F}-$ G): Representative PAGE gel and summarized data show the CAT activity, Representative Western blot gel and summarized data show the protein of Ki67, $\mathrm{Y}-\mathrm{H} 2 \mathrm{AX}$, p21 expression in the nucleus, CD31, VWF expression on the cell cytomembrane, $\mathrm{H} 3$ and pan-cadherin as the normalization of nucleus protein and cytomembrane protein respectively (the full-length gels were showed in supplemental figure 2-C); ( $\mathrm{H}-\mathrm{I})$ : Summarized data show hydrogen peroxide accumulation in EPC. ${ }^{*} p<0.05$ vs. Control (Ctrl); $\# p<0.05$ vs. HG or LPS+ATP treated group $(n=3)$. 


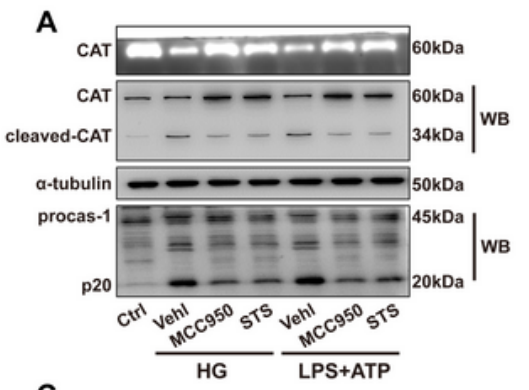

C
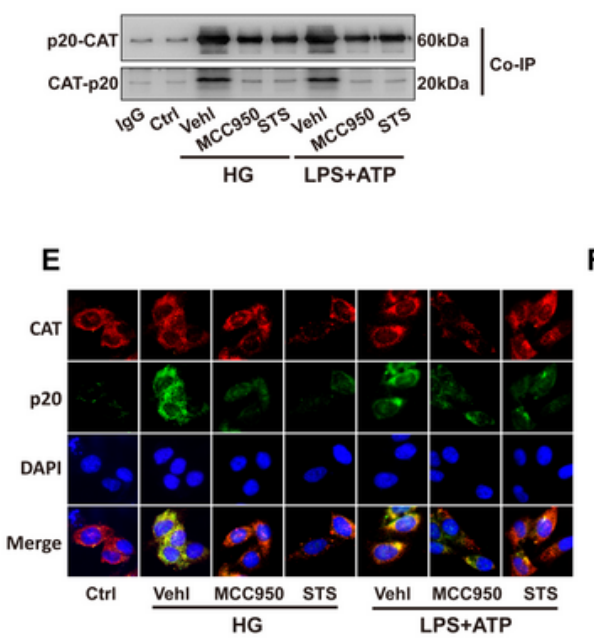

$$
\text { G }
$$

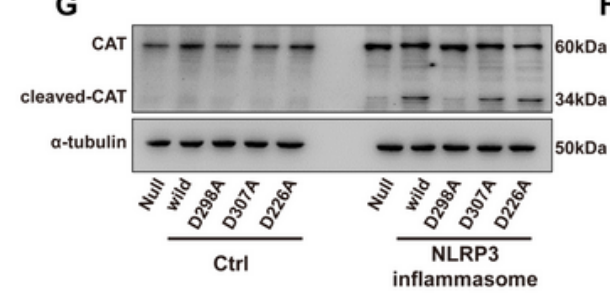

$$
\text { I }
$$

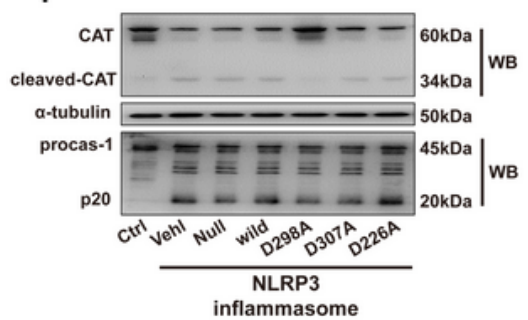

K

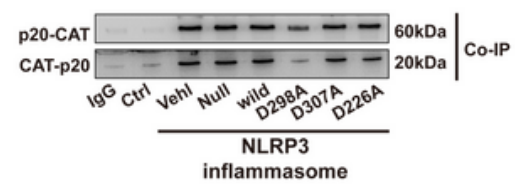

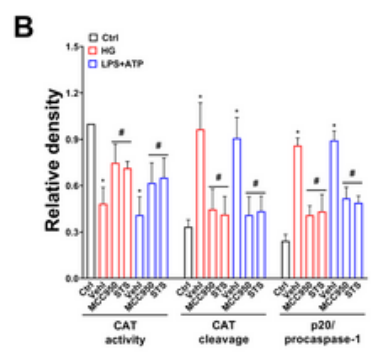
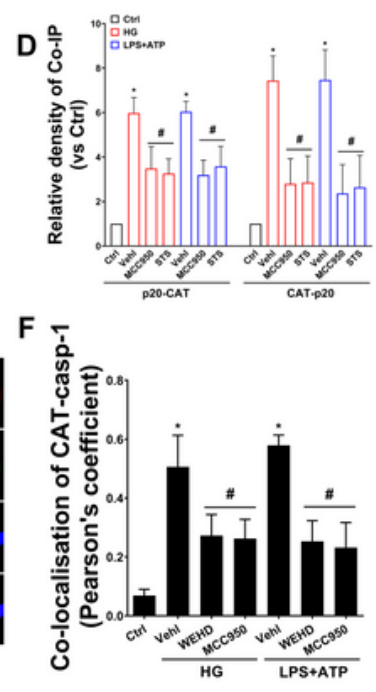

H
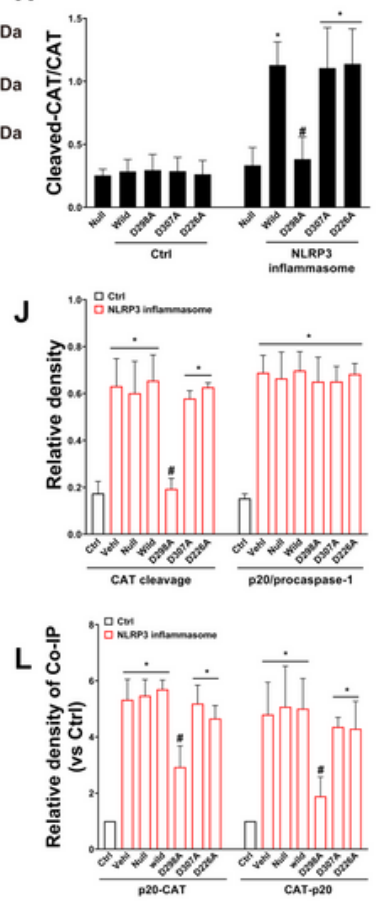

\section{Figure 4}

STS protected CAT activity by inhibiting NLRP3 inflammasome dependent caspase-1 activation (A-B): Representative PAGE gel and summarized data show the CAT activity, representative Western blot gel and summarized data show and the degree of CAT cleavage and p20 production (the full-length gels were showed in supplemental figure 2-D); (C-D): Representative Co-immunoprecipitation gel and summarized data show the p20-CAT and CAT-p20 coprecipitation; (E-F): Representative immunofluorescence images 
(200x) and summarized coefficient of co-localization of CAT and p20; (G-H): Representative Western blot gel and summarized data show the degree of CAT cleavage in aspartic acid mutated EPC; (I-J):

Representative Western blot gel and summarized data show the degree of CAT cleavage and p20 production in aspartic acid mutated EPC; $(\mathrm{K}-\mathrm{L})$ : Representative Co-immunoprecipitation gel and summarized data show the p20-CAT and CAT-p20 coprecipitation. ${ }^{*} p<0.05$ vs. Control (Ctrl); $\# p<0.05$ vs. HG or LPS+ATP treated group or NLRP3 inflammasome plasmids transfected and activated group $(n=3)$.
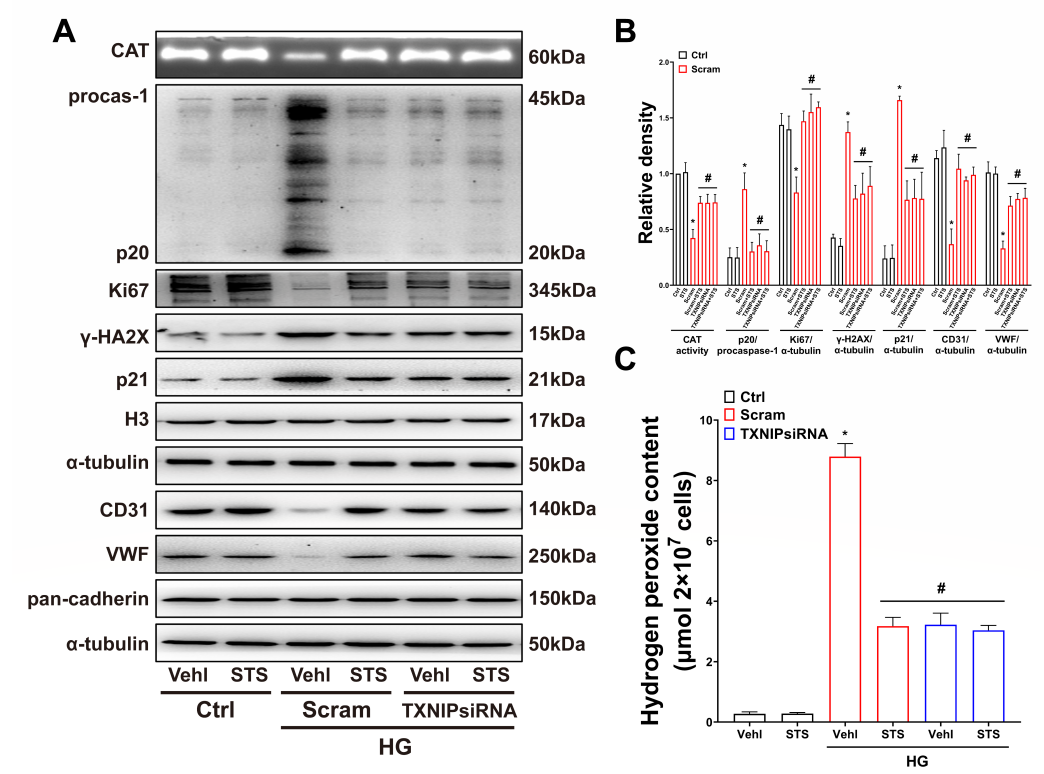

D
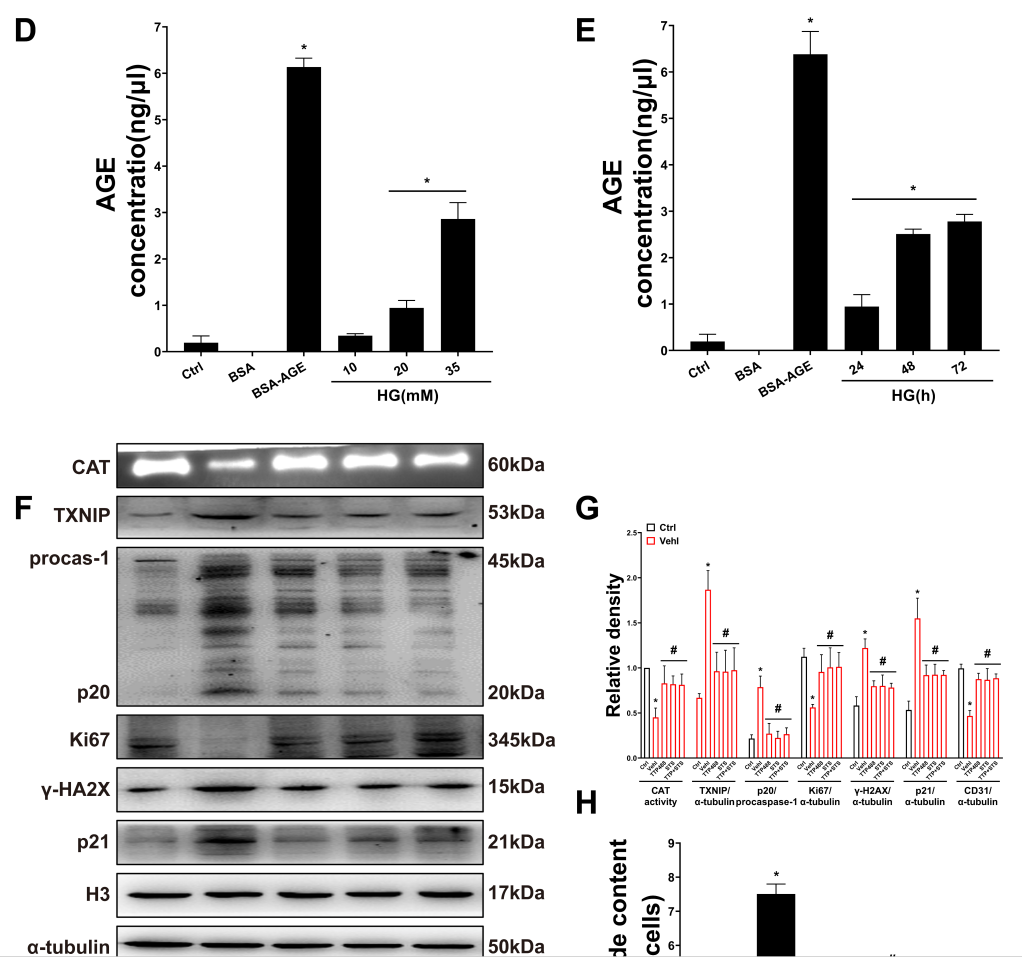

Figure 5 
STS improved CAT activity and EPC function by inhibiting the RAGE-TXNIP-caspase-1 pathway (A-B): Representative PAGE gel and summarized data show the CAT activity, Representative Western blot gel and summarized data show the protein of Ki67, $\mathrm{Y}-\mathrm{H} 2 \mathrm{AX}$, p21 expression in the nucleus, CD31, VWF expression on the cell cytomembrane, $\mathrm{H} 3$, and pan-cadherin as the normalization of nucleus protein and cytomembrane protein respectively (the full-length gels were showed in supplemental figure 2-E); (C): Summarized data show hydrogen peroxide accumulation in EPC; (D-E): Summarized data show AGE content in EPC culture supernatant; (F-G): Representative PAGE gel and summarized data show the CAT activity, Representative Western blot gel and summarized data show the protein of Ki67, y-H2AX, p21 expression in the nucleus, CD31, VWF expression on the cell cytomembrane, $\mathrm{H} 3$ and pan-cadherin as the normalization of nucleus protein and cytomembrane protein respectively (the full-length gels were showed in supplemental figure 2-E); (H): Summarized data show hydrogen peroxide accumulation in EPC. ${ }^{*} p<0.05$ vs. Control (Ctrl); $\# p<0.05$ vs. HG or BSA-AGE treated group $(n=3)$. 

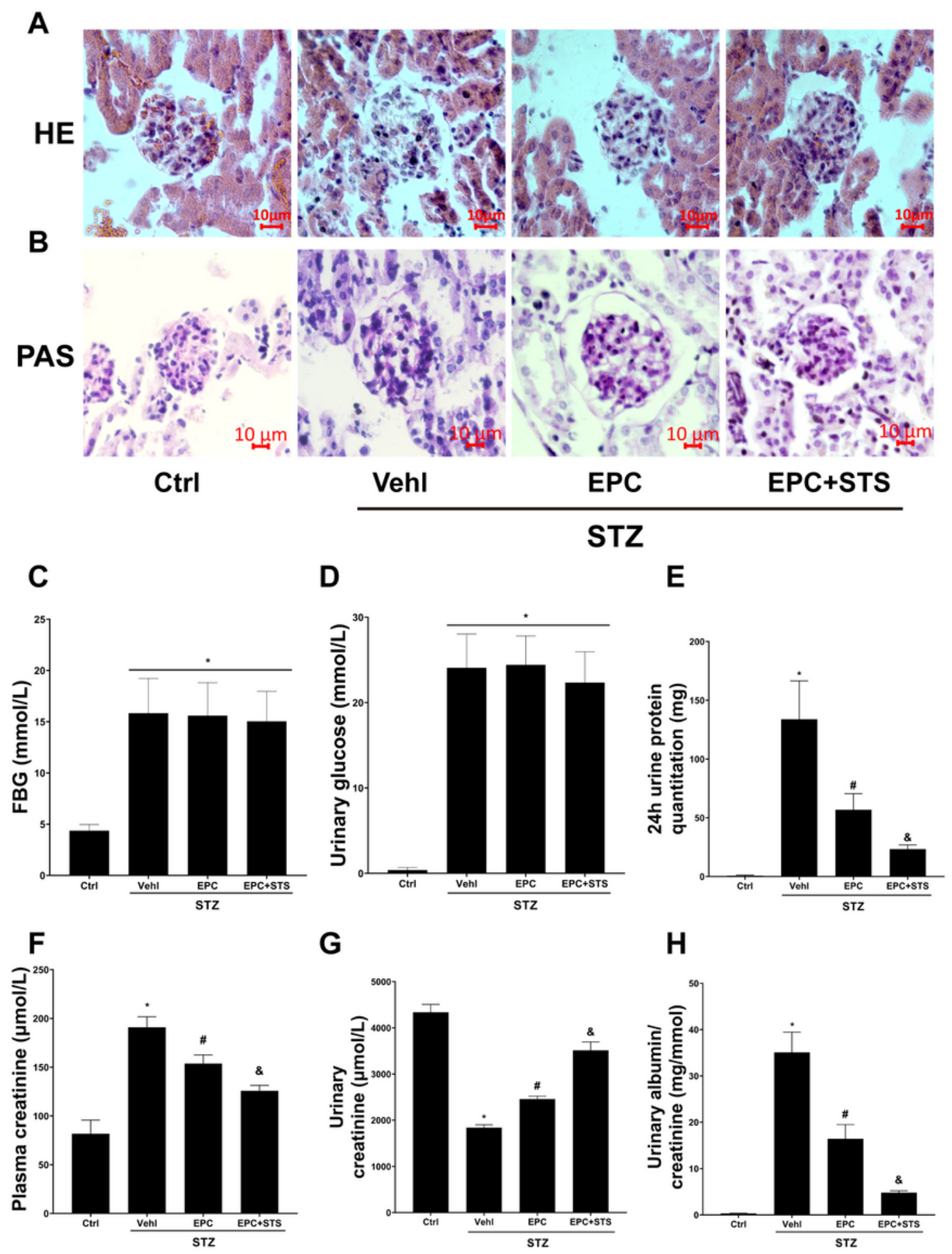

G

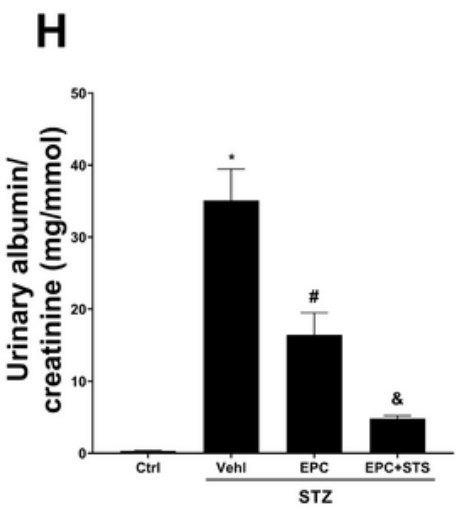

Figure 6

STS enhanced EPC therapy of ameliorating renal function in diabetic mice (A-B): Representative images of HE and PAS staining morphological change; (C): Summarized data of fasting blood glucose; (D): Summarized data of urinary glucose; (E): Summarized data of 24h urine protein quantitation; $(F)$ : Summarized data of plasma creatinine; $(G)$ : Summarized data of urinary creatinine; $(H)$ : Summarized 
data of urinary albumin/creatinine. ${ }^{*} p<0.05$ vs. Control (Ctrl); $\# p<0.05$ vs. STZ treated group; $\& p<0.05$ vs. STZ treated group with EPC transplantation $(n=6)$.

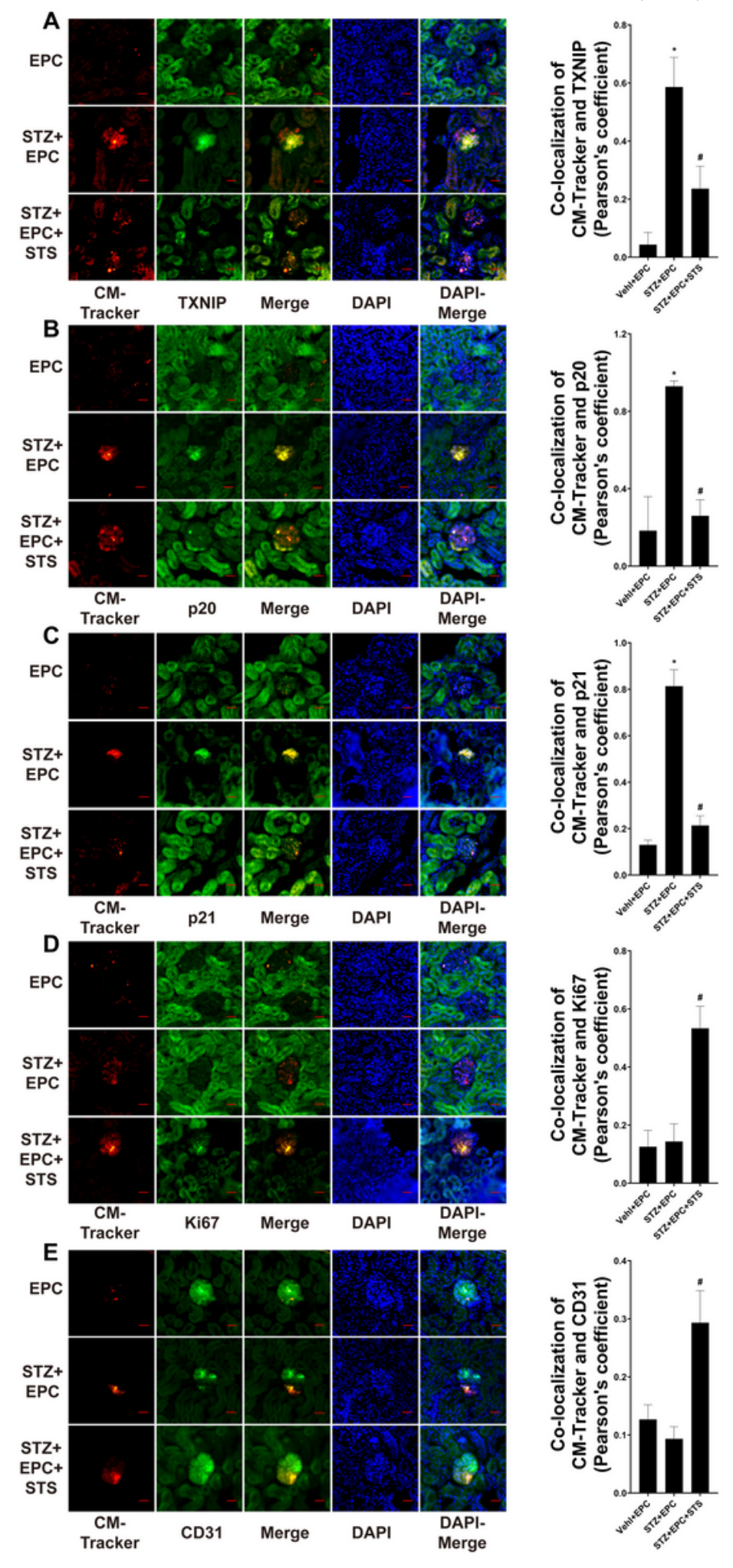

Figure 7

STS protected EPC function in diabetic mice glomeruli Representative immunofluorescence images (200x) and summarized coefficient of colocalization of CM-Tracker labeled EPC and TXNIP (A), p20 (B), p21 (C), Ki67 (D), CD31 (E) in nude mice glomeruli; * $p<0.05$ vs. EPC; \#p<0.05 vs. STZ+EPC ( $=6)$. 


\section{Supplementary Files}

This is a list of supplementary files associated with this preprint. Click to download.

- weiweisupplementalfigure1.tif

- weiweisupplementalfigure2.tif

- weiweisupplementalmanuscript.docx 